MATHEMATICS OF COMPUTATION

Volume 80, Number 276, October 2011, Pages 1949-1978

S $0025-5718(2011) 02481-6$

Article electronically published on April 1, 2011

\title{
FINITE ELEMENT APPROXIMATIONS IN A NON-LIPSCHITZ DOMAIN: PART II
}

\author{
GABRIEL ACOSTA AND MARÍA G. ARMENTANO
}

\begin{abstract}
In a paper by R. Durán, A. Lombardi, and the authors (2007) the finite element method was applied to a non-homogeneous Neumann problem on a cuspidal domain $\Omega \subset \mathbb{R}^{2}$, and quasi-optimal order error estimates in the energy norm were obtained for certain graded meshes. In this paper, we study the error in the $L^{2}$ norm obtaining similar results by using graded meshes of the type considered in that paper. Since many classical results in the theory Sobolev spaces do not apply to the domain under consideration, our estimates require a particular duality treatment working on appropriate weighted spaces.

On the other hand, since the discrete domain $\Omega_{h}$ verifies $\Omega \subset \Omega_{h}$, in the above-mentioned paper the source term of the Poisson problem was taken equal to 0 outside $\Omega$ in the variational discrete formulation. In this article we also consider the case in which this condition does not hold and obtain more general estimates, which can be useful in different problems, for instance in the study of the effect of numerical integration, or in eigenvalue approximations.
\end{abstract}

\section{INTRODUCTION}

The finite element method has been widely studied in several contexts involving different kinds of differential equations; however, the domains under consideration are in general polygons or smooth domains. In the recent paper [2, the piecewise linear finite element method was applied to a non-homogeneous Poisson problem in a domain with an external cusp. Despite its simplicity, this problem provides an interesting starting point for the finite element analysis of more general equations in non-Lipschitz domains. These kinds of problems have interesting applications in fluid mechanics. For instance, the motion of rigid bodies immersed in a fluid can lead to the presence of cusps as a result of collisions between bodies or between a body and the boundary [18, 22.

An interesting feature related with problems in this kind of domain is that even regular solutions may require some type of mesh adaptivity. Indeed, as it was proved in [1, the solution of the proposed problem belongs to $H^{2}$ and, despite of this fact, uniform meshes show a poor convergence rate. The reason for this behavior is related to the fact that, in this context, classical extension theorems do not apply [20, 23]. A solution for this drawback was also given in [2] and

Received by the editor April 6, 2009 and, in revised form, September 13, 2010.

2010 Mathematics Subject Classification. Primary 65N30, 46 E35.

Key words and phrases. Cuspidal domains, finite elements, graded meshes.

This work was supported by ANPCyT under grants PICT 2006-01307 and PICT-2007-00910, and by the Universidad de Buenos Aires under grant X007 and by CONICET under grant PIP $5478 / 1438$. 
(quasi) optimal convergence order error in the energy norm was recovered by using appropriate mesh adaptivity. Let us notice that, for problems in polygonal domains with solutions having corner-like singularities, the use of graded meshes has been widely studied (see [8, 11, 12, 15] and the references therein) and optimal or quasioptimal convergence rates for numerical approximations are usually obtained by using arguments based on weighted Sobolev spaces.

In this paper we continue the analysis of the finite element method for the same problem considered in [2], focusing on $L^{2}$ convergence results. These estimates require a particular treatment, making it necessary to take into account the regularity of the extended functions outside the non-Lipschitz domain under consideration. We introduce two different kinds of auxiliary problems: one in the original domain $\Omega$, and the other in the discrete domain $\Omega_{h}$. The first one leads us to standard estimates of the error in $\Omega$, and the second one to estimates of the error between a certain extension of the original solution and the discrete solution in $\Omega_{h}$. The latter case is more general than the former, however, it is also much more technical and relies on certain extra assumptions. In both cases quasi-optimal order of convergence with respect to the number of nodes is obtained by using appropriate graded meshes of the type considered in [2]. We present some numerical examples supporting this analytical result, and in particular we show that uniform meshes lead to poor $L^{2}$ convergence order (similar conclusions for the $H^{1}$ norm were obtained in [2]).

Let $\Omega \subset \mathbb{R}^{2}$ be defined by

$$
\Omega=\left\{(x, y): 0<x<1,0<y<x^{\alpha}\right\},
$$

where $\alpha>1$. We denote the boundary of $\Omega$ by $\Gamma=\Gamma_{1} \cup \Gamma_{2} \cup \Gamma_{3}$, where

$\Gamma_{1}=\{0 \leq x \leq 1, y=0\}, \quad \Gamma_{2}=\{x=1,0 \leq y \leq 1\}$ and $\Gamma_{3}=\left\{0 \leq x \leq 1, y=x^{\alpha}\right\}$

(see Figure 1).

Our model problem is: find $u$ such that

$$
\left\{\begin{aligned}
-\Delta u & =f, & & \text { in } \Omega, \\
\frac{\partial u}{\partial \nu} & =g, & & \text { on } \Gamma_{3}, \\
\frac{\partial u}{\partial \nu} & =0, & & \text { on } \Gamma_{1}, \\
u & =0, & & \text { on } \Gamma_{2},
\end{aligned}\right.
$$

where $\nu$ denotes the outside normal.

We will work along this paper with $g=0$. This assumption partially simplifies the treatment of the error and can be justified by recalling that $H^{2}$ regularity results for (1.1) rely on the smoothness of $g$ and its fast decay to zero, i.e., $g \sim 0$ near the tip of the cusp [1].

Let $V=\left\{v \in H^{1}(\Omega):\left.v\right|_{\Gamma_{2}}=0\right\}$. Then, the variational formulation of our model problem (1.1) is given by: find $u \in V$ such that

$$
a(u, v)=L(v) \quad \forall v \in V
$$

where $a(u, v)=\int_{\Omega} \nabla u \cdot \nabla v$ and $L(v)=\int_{\Omega} f v$. It is known that this problem has a unique solution in $H^{2}(\Omega)$ and that there exists a constant $C$ such that (see [1, 15, 19])

$$
\|u\|_{H^{2}(\Omega)} \leq C\|f\|_{L^{2}(\Omega)}
$$




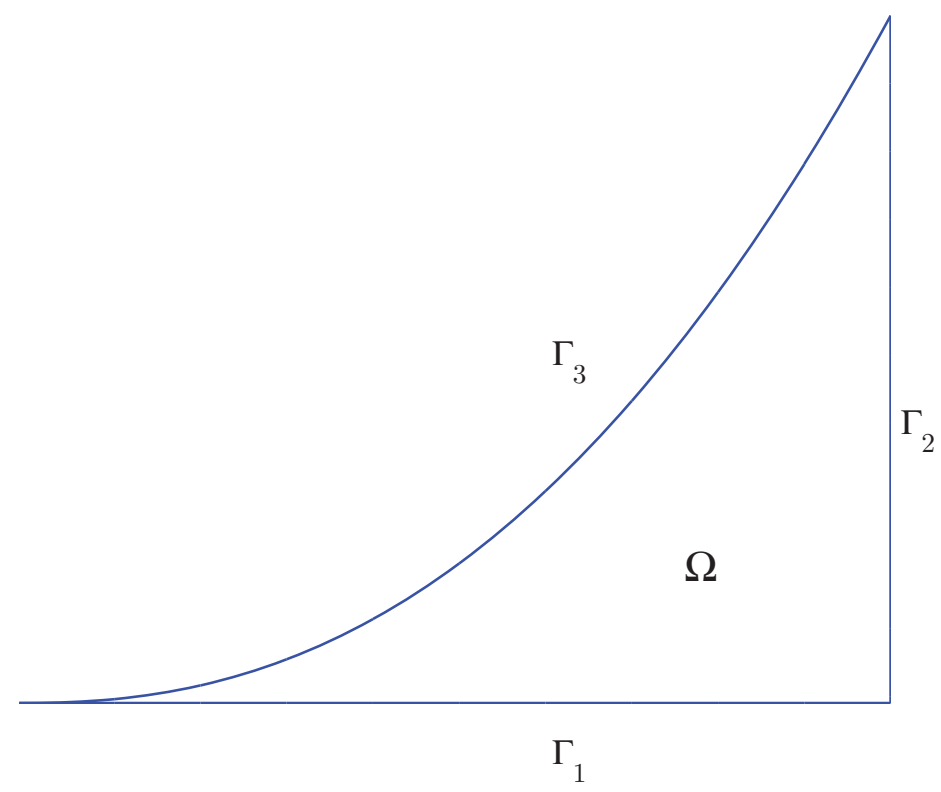

Figure 1. Cuspidal domain

The natural way to approximate the solution of (1.2) is to replace $\Omega$ by a polygonal domain $\Omega_{h}$ and then use the standard finite element method. We will construct $\Omega_{h}$ in such a way that $\Omega \subset \Omega_{h}$ and the nodes of $\Gamma_{h}$, the boundary of $\Omega_{h}$, are on $\Gamma$.

Let $\left\{\mathcal{T}_{h}\right\}$ be a family of triangulations of $\Omega_{h}$ verifying the maximum angle condition. We can associate to $\left\{\mathcal{T}_{h}\right\}$ the finite element space

$$
V_{h}=\left\{v \in H^{1}\left(\Omega_{h}\right):\left.v\right|_{\Gamma_{2}}=0 \text { and }\left.v\right|_{T} \in \mathcal{P}_{1} \quad \forall T \in \mathcal{T}_{h}\right\},
$$

where $\mathcal{P}_{1}$ denotes the space of linear polynomials.

Then, the finite element approximation problem of (1.2) is: find $u_{h} \in V_{h}$ such that

$$
a_{h}\left(u_{h}, v_{h}\right)=L_{h}\left(u_{h}\right) \quad \forall v_{h} \in V_{h},
$$

where $a_{h}(u, v)=\int_{\Omega_{h}} \nabla u \cdot \nabla v$ and $L_{h}(v)=\int_{\Omega_{h}} f v$. Observe that the discrete problem corresponds to a boundary problem on $\Omega_{h}$.

Since the solution of problem (1.1) depends on the values of $f$ in $\Omega$ only, it seems natural to assume that $f$ vanishes outside $\Omega$, in which case we have $L_{h}(v)=\int_{\Omega} f v$, and so (1.4) agrees with the discrete problem from [2]. In this paper we also consider the case in which this assumption is dropped and obtain more general error estimates for finite element approximations. This approach introduces an extra difficulty that was not addressed in 2]; however, it provides more information in different scenarios. Indeed, the contribution of terms such as $\int_{\Omega_{h} \backslash \Omega} \tilde{f} v_{h}$, with $\tilde{f}$ being a certain approximation of $f$ defined on $\Omega_{h}$, can be useful to evaluate the effect of numerical integration (see, for example, 24]). Moreover, since $\Omega \neq \Omega_{h}$, the standard theory for eigenvalue approximations [9] does not apply straightforwardly. In fact, the study of convergence for this problems leads to problems such as (1.4) with $f$ not necessarily equal to zero outside $\Omega$ [17, 24]. On the other hand, the 
study of the error between a certain extension of the solution $u$ and $u_{h}$, analyzed in Section 5, is also of interest in the context of eigenvalue approximations [17, 24].

Let us mention that, even when $\Omega$ is not regular enough, certain extension operators can be constructed. More precisely, the solution of (1.2) can be extended to a function in a weighted Sobolev space, with the weight being a power of the distance to the cuspidal point (see [2, 20]). In fact, there exists a function $u^{E} \in H_{\alpha}^{2}\left(\mathbb{R}^{2}\right)$ such that $\left.u^{E}\right|_{\Omega}=u$, and

$$
\left\|u^{E}\right\|_{H_{\alpha}^{2}\left(\mathbb{R}^{2}\right)} \leq C\|u\|_{H^{2}(\Omega)},
$$

where the weighted Sobolev space $H_{\alpha}^{2}$ is defined, for any domain $\mathcal{D} \subset \mathbb{R}^{2}$, as follows:

$$
H_{\alpha}^{2}(\mathcal{D})=\left\{v: r^{\frac{\alpha-1}{2}} D^{\delta} v \in L^{2}(\mathcal{D}) \quad \forall \delta,|\delta| \leq 2\right\}
$$

with $r=\sqrt{x^{2}+y^{2}}$, and

$$
\|v\|_{H_{\alpha}^{2}(\mathcal{D})}^{2}=\sum_{|\delta| \leq 2}\left\|r^{\frac{\alpha-1}{2}} D^{\delta} v\right\|_{L^{2}(\mathcal{D})}^{2} .
$$

This extension result will be useful to bound the approximation error in $L^{2}$.

The rest of the paper is organized as follows. In Section 2 we present some results involving the graded meshes that we will use in the remainder of the paper. In Section 3 we obtain $L^{2}$ error estimates in $\Omega$ when $f \equiv 0$ outside $\Omega$. Section 4 is devoted to obtaining $H^{1}$ and $L^{2}$ error estimates when $f$ is not necessarily equal to zero outside $\Omega$ in the discrete variational formulation. In Section 5 we introduce and analyze an auxiliary problem on $\Omega_{h}$, which is the main tool to obtain $L^{2}$ error estimates in $\Omega_{h}$ between $u_{h}$ and a certain extension of $u$. Finally, in Section 6 we explain how the graded meshes can be constructed, and we present numerical approximations in which the error behaves according to our analytical results.

\section{Graded Meshes}

We will assume that the family of meshes $\left\{\mathcal{T}_{h}\right\}$ satisfies the same properties as those considered in [2]. More precisely, we take $1<\alpha<3$ and define $\gamma=(\alpha-1) / 2$. Let $\mathcal{T}_{h}$ be a triangulation of $\Omega_{h}$, where $\Omega_{h}$ is an approximate polygon of $\Omega$ with all its vertices belonging to $\Gamma$, and let $h>0$ be a parameter that goes to 0 . If for each $T \in \mathcal{T}_{h}$ we denote by $h_{T}$ its diameter and by $\theta_{T}$ its maximum angle, we assume that there exist positive constants $\sigma$ and $\theta_{M}<\pi$, independent of $h$, such that

(1) $\theta_{T}<\theta_{M}, \forall T \in \mathcal{T}_{h}$ (the maximum angle condition).

(2) $h_{T} \sim \sigma h^{\frac{1}{1-\gamma}}$, if $(0,0) \in T$.

(3) $h_{T} \leq \sigma h \inf _{T} x^{\gamma}$, if $(0,0) \notin T$.

We denote by $\Gamma_{3, h}^{j}, 1 \leq j \leq n$, the edges on the boundary of $\Omega_{h}$, by $P_{j-1}=$ $\left(x_{j-1}, x_{j-1}^{\alpha}\right)$ and $P_{j}=\left(x_{j}, x_{j}^{\alpha}\right)$ their endpoints with $x_{0}=0$ and $x_{n}=1$, and by $\Gamma_{3}^{j}$ the part on $\Gamma_{3}$ with the same endpoints (see Figure 2). By $\Omega_{h}^{j}$ we denote the region bounded by $\Gamma_{3}^{j}$ and $\Gamma_{3, h}^{j}$.

In addition to the assumptions (1), (2) and (3) we will need for our error analysis the following hypothesis on the meshes:

(Ha) For $1 \leq j \leq n$ the region $\Omega_{h}^{j}$ is strictly contained in only one triangle denoted by $T_{j}$. We denote the diameter of $T_{j}$ by $h_{j}$. 


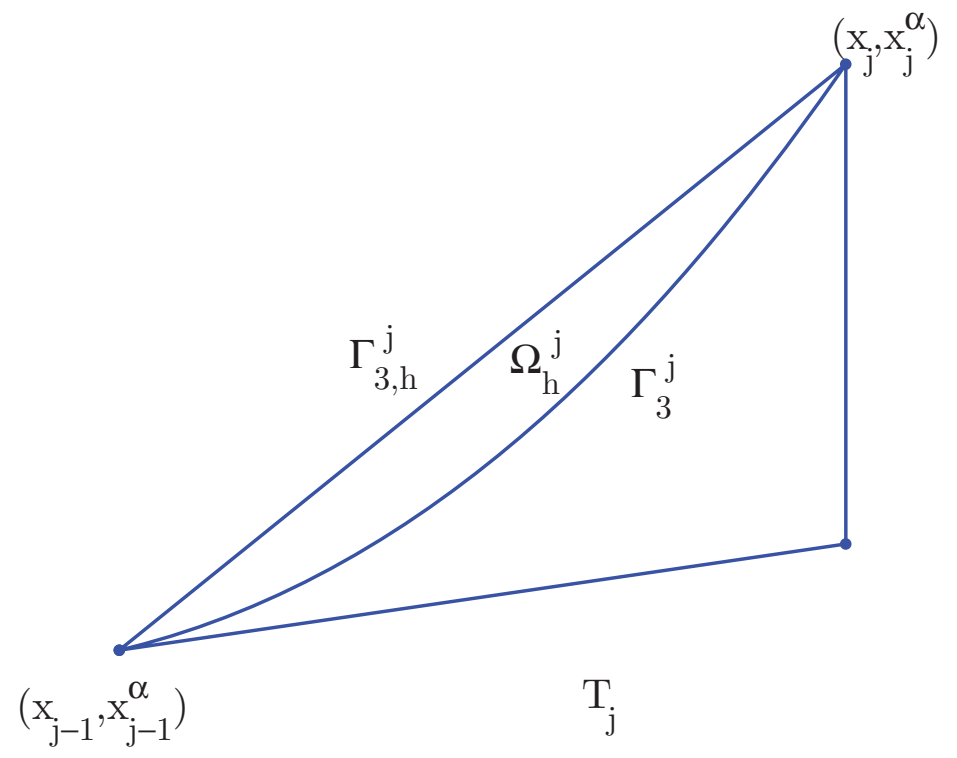

FiguRE 2

Let us also notice that, for $2 \leq j \leq n$,

$$
x_{j} \leq C x_{j-1},
$$

where $C$ can be taken independent of $h$. Indeed, from (Ha) we have $x_{j}-x_{j-1} \leq$ $C\left|\Gamma_{3, h}^{j}\right|$ for some constant depending only on $\alpha$. Then $x_{j}-x_{j-1} \leq C h_{j}$, and hence from assumption (3) we get

$$
x_{j} \leq x_{j-1}\left(1+C h x_{j-1}^{\gamma-1}\right) .
$$

Therefore, we have proved the following useful result.

Lemma 2.1. For $2 \leq j \leq n$,

$$
x_{j-1} \leq x_{j} \leq C x_{j-1},
$$

with $C$ depending only on $\alpha$ and $\sigma$.

Remark 2.1. We will show in Section 6 that meshes verifying conditions (1), (2), (3) and (Ha) can indeed be constructed.

We will assume that our family of triangulations satisfies conditions (1), (2), (3), and hypothesis (Ha). The following result is obtained in the proof of Lemma 4.1 in 2]; we reproduce it here as a separate result for the sake of completeness.

Lemma 2.2. Let $\gamma=\frac{\alpha-1}{2}$ with $1<\alpha<3$, and choose $0<\beta$ and $q>1$ such that

$$
\beta q<\min \{2 \gamma, 1\} .
$$

Then

$$
\int_{\Omega_{h} \backslash \Omega} x^{-2 \beta q} \leq C h^{2} .
$$


Proof.

$$
\int_{\Omega_{h} \backslash \Omega} x^{-2 \beta q}=\sum_{j=1}^{N} \int_{\Omega_{h}^{j}} x^{-2 \beta q} .
$$

Let us estimate each term on the right hand side of (2.7). Since

$$
\Omega_{h}^{1} \subset T=\left\{(x, y): 0 \leq x \leq x_{1}, 0 \leq y \leq x_{1}^{\alpha-1} x\right\},
$$

we have

$$
\int_{\Omega_{h}^{1}} x^{-2 \beta q} \leq \int_{T} x^{-2 \beta q} .
$$

Hence, using now that $h_{1} \leq \sigma h^{\frac{1}{1-\gamma}}$ and $\beta q<1$, we obtain

$$
\int_{T} x^{-2 \beta q} \leq C h_{1}^{2(\gamma+1-\beta q)} \leq C h^{2 \frac{\gamma+1-\beta q}{1-\gamma}}
$$

and, therefore,

$$
\int_{T} x^{-2 \beta q} \leq C h^{2}
$$

because $\beta q<2 \gamma$.

On the other hand, we have

$$
\sum_{j>1} \int_{\Omega_{h}^{j}} x^{-2 \beta q} \leq \sum_{j>1} x_{j-1}^{-2 \beta q}\left|\Omega_{h}^{j}\right|,
$$

but, by using the well known error formula for the trapezoidal rule, we obtain

$$
\left|\Omega_{h}^{j}\right| \leq C h_{j}^{3} x_{j-1}^{\alpha-2}=C h_{j}^{3} x_{j-1}^{2 \gamma-1},
$$

where in the case when $\alpha>2$ we have used $x_{j} \leq C x_{j-1}$. Therefore, since $h_{j} \leq$ $\sigma h x_{j-1}^{\gamma}$ we have

$$
\begin{aligned}
\sum_{j>1} \int_{\Omega_{h}^{j}} x^{-2 \beta q} & \leq C \sum_{j>1} x_{j-1}^{-2 \beta q+2 \gamma-1} h_{j}^{3} \leq C h^{2} \sum_{j>1} x_{j-1}^{-2 \beta q+4 \gamma-1} h_{j} \\
& \leq C h^{2} \int_{0}^{1} x^{-2 \beta q+4 \gamma-1}
\end{aligned}
$$

where we have used again that $x_{j} \leq C x_{j-1}$. But the last integral is finite because $\beta q<2 \gamma$.

We will also need bounds for the measure of the set $\Omega_{h} \backslash \Omega$ in terms of the parameter $h$.

Lemma 2.3. It holds that

$$
\left|\Omega_{h} \backslash \Omega\right| \leq C h^{2} .
$$

Proof. It follows by using similar arguments as those in the proof of Lemma 2.2, or as a corollary, taking into account that $1 \leq x^{-2 \beta q}$, where $\beta$ and $q$ are as in the previous lemma.

In order to obtain $L^{2}$ error estimates in the polygonal domains $\Omega_{h}$ we will need a careful estimate for the inner angles of $\Omega_{h}$. This computation is carried out in the following lemma.

Lemma 2.4. Let $\omega_{h}$ be the maximum inner angle of $\Omega_{h}$. Then:

i) if $\alpha<2, \quad \omega_{h} \leq \pi+C \alpha(\alpha-1) h^{\frac{\alpha-1}{3-\alpha}}$, 
ii) if $2 \leq \alpha<3, \quad \omega_{h} \leq \pi+C \alpha(\alpha-1) h$, where $C$ is independent of $\alpha$ and $h$.

Proof. Assume first that $j \geq 2$ (the case $j=1$ is treated below). If we denote by $\omega_{h, j}$ the inner angle between $\Gamma_{3, h}^{j}$ and $\Gamma_{3, h}^{j+1}$, we obviously have

$$
\omega_{h, j}=\pi+\arctan \left(\frac{x_{j+1}^{\alpha}-x_{j}^{\alpha}}{x_{j+1}-x_{j}}\right)-\arctan \left(\frac{x_{j}^{\alpha}-x_{j-1}^{\alpha}}{x_{j}-x_{j-1}}\right) .
$$

But

$$
\frac{x_{j+1}^{\alpha}-x_{j}^{\alpha}}{x_{j+1}-x_{j}}=\alpha \tilde{x}_{j+1}^{\alpha-1}, \quad \frac{x_{j}^{\alpha}-x_{j-1}^{\alpha}}{x_{j}-x_{j-1}}=\alpha \tilde{x}_{j}^{\alpha-1}
$$

for some $\tilde{x}_{j+1} \in\left[x_{j}, x_{j+1}\right] \tilde{x}_{j} \in\left[x_{j-1}, x_{j}\right]$, and using the fact that the function $\arctan \left(\alpha t^{\alpha-1}\right)$ is increasing, we get

$$
\omega_{h, j} \leq \pi+\arctan \left(\alpha x_{j+1}^{\alpha-1}\right)-\arctan \left(\alpha x_{j-1}^{\alpha-1}\right) .
$$

By the mean value theorem and Lemma 2.1

$$
\begin{aligned}
\arctan \left(\alpha x_{j+1}^{\alpha-1}\right)-\arctan \left(\alpha x_{j-1}^{\alpha-1}\right) & =C \frac{\alpha(\alpha-1) x_{j}^{\alpha-2}}{1+\alpha^{2} x_{j}^{2(\alpha-1)}}\left(x_{j+1}-x_{j-1}\right) \\
& \leq C \alpha(\alpha-1) x_{j}^{\alpha-2}\left(x_{j+1}-x_{j-1}\right) \\
& \leq C h \alpha(\alpha-1) x_{j}^{\alpha-2} x_{j}^{\frac{\alpha-1}{2}}
\end{aligned}
$$

where in the last inequality we have used condition (3).

Now, if $\alpha \geq 2$, the result follows immediately. For the case $\alpha<2$ we use the fact that $x_{j} \geq x_{1}$ and that, by condition $(2), x_{1} \sim h^{\frac{2}{3-\alpha}}=h^{\frac{1}{1-\gamma}}$, so

$$
\arctan \left(\alpha x_{j+1}^{\alpha-1}\right)-\arctan \left(\alpha x_{j-1}^{\alpha-1}\right) \leq C \alpha(\alpha-1) h^{\frac{2(\alpha-2)}{3-\alpha}+1}=C \alpha(\alpha-1) h^{\frac{\alpha-1}{3-\alpha}},
$$

obtaining the desired result.

Let us now focus on the case $j=1$. In this case (2.8) takes the form

$\omega_{h, 1}=\pi+\arctan \left(\frac{x_{2}^{\alpha}-x_{1}^{\alpha}}{x_{2}-x_{1}}\right)-\arctan \left(x_{1}^{\alpha-1}\right)=\pi+\arctan \left(\alpha \tilde{x}_{2}^{\alpha-1}\right)-\arctan \left(x_{1}^{\alpha-1}\right)$

with $\tilde{x}_{2} \in\left[x_{1}, x_{2}\right]$, but

so

$$
x_{1}^{\alpha-1}=\alpha\left(\frac{x_{1}}{\alpha^{1 /(\alpha-1)}}\right)^{\alpha-1}=\alpha \tilde{x}_{1}^{\alpha-1}
$$

$$
\tilde{x}_{1} \leq x_{1} \leq C \tilde{x}_{1}
$$

with $C$ depending only on $\alpha$. Then

$$
\omega_{h, 1} \leq \pi+\arctan \left(\alpha \tilde{x}_{2}^{\alpha-1}\right)-\arctan \left(\alpha \tilde{x}_{1}^{\alpha-1}\right),
$$

and the result now follows as in the case $j \geq 2$ using (2.9).

In Theorem 2.4 from [5, page 63] T. Apel obtained interpolation error estimates for functions in weighted Sobolev spaces on tetrahedral elements under the maximum angle condition. However, we were unable to find analogous results for the two dimensional case. The reason for this seems to be that corner singularities, which lead to the kind of spaces considered in this work, do not require anisotropic elements in the case of polygonal domains (see, for instance, [15]). In our case, the external cusp enforces the occurrence of flat elements, and, hence, we need to 
obtain the required error estimates for functions in $H_{\alpha}^{2}$ in dimension 2 under the maximal angle condition. In order to do that, we prove the following Poincaré type inequality for functions with zero average on a side of a triangle.

Lemma 2.5. Let $\hat{T}$ be the following "reference" triangle, $\hat{T}=\left\{(x, y) \in \mathbb{R}^{2}: 0 \leq\right.$ $x \leq 1,0 \leq y \leq x\}$, and $w$ be such that $\|w\|_{L^{2}(\hat{T})}+\left\|\nabla w x^{s}\right\|_{L^{2}(\hat{T})}<\infty$ for some $0 \leq s<1$. If $\int_{\ell} w=0$ where $\ell$ is a side of $\hat{T}$, then there exists a positive constant $C$, depending only on $s$ and $\hat{T}$, such that

$$
\|w\|_{L^{2}(\hat{T})} \leq C\left\|\nabla w x^{s}\right\|_{L^{2}(\hat{T})} .
$$

Proof. We observe that for any $0 \leq s<1$, there exists $p>1$ such that

$$
\|v\|_{L^{p}(\hat{T})} \leq C\left\|v x^{s}\right\|_{L^{2}(\hat{T})} .
$$

Indeed, since

$$
\int_{\hat{T}}|v|^{p}=\int_{\hat{T}}|v|^{p} x^{p s} x^{-p s}
$$

by applying Hölder's inequality with exponent $\frac{2}{p}$ and its dual exponent $\frac{2}{2-p}$ we obtain (2.10) for any $p$ such that $p<\frac{2}{s+1}$. On the other hand, it is easy to see by standard compactness arguments (see Lemma 2.2 in [3] for the case $p=2$ ) that functions with zero average on one side of $\hat{T}$ verify

$$
\|w\|_{L^{p}(\hat{T})} \leq C\|\nabla w\|_{L^{p}(\hat{T})},
$$

with $C$ depending only on $p$ and $\hat{T}$. Therefore,

$$
\|w\|_{L^{2}(\hat{T})} \leq C\|w\|_{W^{1, p}(\hat{T})} \leq C\|\nabla w\|_{L^{p}(\hat{T})} \leq C\left\|\nabla w x^{s}\right\|_{L^{2}(\hat{T})} .
$$

Indeed, the first inequality follows by the classical embedding theorem, while the second and third inequalities are consequences of (2.11) and (2.10) with $v=\nabla w$.

Theorem 2.1. Let $T$ be a triangle with a maximum interior angle $\theta_{T}$, and let $\mathbf{v}_{m}$ be the vertex corresponding to the minimum interior angle of $T$. We denote by $d_{\mathbf{v}_{m}}(x, y)$ the distance from $(x, y) \in T$ to $\mathbf{v}_{m}$. Let $v$ be such that $\|v\|_{L^{2}(T)}+$ $\|\nabla v\|_{L^{2}(T)}+\sum_{|\delta|=2}\left\|D^{\delta} v d_{\mathbf{v}_{m}}^{s}\right\|_{L^{2}(T)}<\infty$ for some $0 \leq s<1$. Then, there exists a positive constant $C$, depending only on $\theta_{T}$, such that

$$
\begin{gathered}
\|\nabla(v-\Pi v)\|_{L^{2}(T)} \leq C h_{T}^{1-s} \sum_{|\delta|=2}\left\|D^{\delta} v d_{\mathbf{v}_{m}}^{s}\right\|_{L^{2}(T)}, \\
\|v-\Pi v\|_{L^{2}(T)} \leq C h_{T}^{2-s} \sum_{|\delta|=2}\left\|D^{\delta} v d_{\mathbf{v}_{m}}^{s}\right\|_{L^{2}(T)},
\end{gathered}
$$

where $\Pi v \in V_{h}$ denotes the piecewise linear Lagrange interpolation of $v$.

Proof. It is clear that it is enough to show (2.12) and (2.13) for a triangle obtained from $T$ after a rigid movement. Hence, we can assume that $T$ is a triangle with $\mathbf{v}_{m}=(0,0)$ and with remaining vertices of the form $\mathbf{v}_{2}=\left(h_{1}, 0\right) \mathbf{v}_{3}=\left(x_{1}, h_{2}\right)$ with $h_{1}, h_{2}>0$ and $h_{1} \geq \sqrt{x_{1}^{2}+h_{2}^{2}} \geq h_{2}$. Therefore, the angle $\theta_{2}$ at $\mathbf{v}_{2}=\left(h_{1}, 0\right)$ verifies $\theta_{2} \leq \pi / 2$ and, since it is not the minimum angle of $T, \theta_{2} \geq \frac{\pi-\theta_{T}}{2}$, i.e.,

$$
\frac{\pi-\theta_{T}}{2} \leq \theta_{2} \leq \pi / 2
$$


Let us introduce a further linear transformation $L$ given by the matrix

$$
A=\left(\begin{array}{cc}
1 & \frac{x_{1}-h_{1}}{h_{2}} \\
0 & 1
\end{array}\right) .
$$

It is clear that $L$ transforms the right triangle $T_{R}$ with vertices $(0,0),\left(h_{1}, 0\right),\left(h_{1}, h_{2}\right)$ into $T$. From (2.14), it is easy to see that $\frac{x_{1}-h_{1}}{h_{2}} \leq C$ for some $C=C\left(\theta_{T}\right)$ and, as a consequence, $\|A\| \leq C$ and $\left\|A^{-1}\right\| \leq C$. Since in both triangles $T$ and $T_{R}$ the minimum angle is placed at the origin, the inequalities $\|(x, y)\| \leq\left\|A^{-1}\right\|\|L(x, y)\| \leq$ $\|A\|\left\|A^{-1}\right\|\|(x, y)\|$ imply the equivalence between the distance $d_{\mathbf{v}_{m}}(x, y)$ and the norm of $L(x, y)$. Therefore, changing variables, we have that it is enough to prove (2.12) and (2.13) for $T_{R}$ with $h_{1} \geq h_{2}$. On the other hand, in $T_{R}$ it is clear that $d_{\mathbf{v}_{m}}=\|(x, y)\| \sim x$, and so in order to show that (2.12) holds it is enough to prove that

$$
\|\nabla(v-\Pi v)\|_{L^{2}\left(T_{R}\right)} \leq C h_{T_{R}}^{1-s} \sum_{|\delta|=2}\left\|D^{\delta} v x^{s}\right\|_{L^{2}\left(T_{R}\right)} .
$$

We prove the previous inequality for $\frac{\partial(v-\Pi v)}{\partial x}$; the other derivative can be treated in the same way. Taking $w=\frac{\partial(v-\Pi v)}{\partial x}$ we have that $\int_{\ell_{1}} w=0$, with $\ell_{1}$ being the side joining the vertices $(0,0)$ and $\left(h_{1}, 0\right)$. Changing variables to the reference element defined in Lemma2.5 we get, taking $\hat{L}(\hat{x}, \hat{y})=\left(\hat{x} h_{1}, \hat{y} h_{2}\right)$, that the function $\hat{w}=w \circ \hat{L}$ has zero average on the side of $\hat{T}$ joining the vertices $(0,0)$ and $(1,0)$. Then, by Lemma 2.5 applied to $\hat{w}$ we get

$$
\|w\|_{L^{2}\left(T_{R}\right)}^{2}=h_{1} h_{2}\|\hat{w}\|_{L^{2}(\hat{T})}^{2} \leq h_{1} h_{2} C\left\|\nabla \hat{w} \hat{x}^{s}\right\|_{L^{2}(\hat{T})}^{2},
$$

and changing variables back to the original $T_{R}$

$$
\|w\|_{L^{2}\left(T_{R}\right)} \leq C\left(h_{1}\left\|\frac{\partial w}{\partial x}\left(\frac{x}{h_{1}}\right)^{s}\right\|_{L^{2}(\hat{T})}+h_{2}\left\|\frac{\partial w}{\partial y}\left(\frac{x}{h_{1}}\right)^{s}\right\|_{L^{2}(\hat{T})}\right)
$$

since $h_{1} \geq h_{2}$,

$$
\|w\|_{L^{2}\left(T_{R}\right)} \leq C h_{T_{R}}^{1-s}\left\|\nabla w x^{s}\right\|_{L^{2}\left(T_{R}\right)}
$$

and (2.15) follows. As is usual when considering anisotropic elements, inequality (2.13) is easier to prove than (2.12), since its left hand side does not involve derivatives. The estimate for $\hat{T}$,

$$
\|\hat{v}-\Pi \hat{v}\|_{L^{2}(\hat{T})} \leq C \sum_{|\delta|=2}\left\|D^{\delta} \hat{v} \hat{x}^{s}\right\|_{L^{2}(\hat{T})},
$$

follows by using embedding results and standard Lagrange interpolation error estimates in $L^{p}$ together with (2.10). In fact,

$$
\|\hat{v}-\Pi \hat{v}\|_{L^{2}(\hat{T})} \leq C\|\hat{v}-\Pi \hat{v}\|_{W^{1, p}(\hat{T})} \leq C \sum_{|\delta|=2}\left\|D^{\delta} \hat{v}\right\|_{L^{p}(\hat{T})} \leq \sum_{|\delta|=2}\left\|D^{\delta} \hat{v} \hat{x}^{s}\right\|_{L^{2}(\hat{T})} .
$$

Now, (2.13) follows on $T_{R}$ from (2.16) by using the change of variables $\hat{L}(\hat{x}, \hat{y})=$ $\left(\hat{x} h_{1}, \hat{y} h_{2}\right)$ and taking into account that $\Pi \hat{v}=\Pi v \circ \hat{L}$.

We define a fixed (i.e., independent of $h$ ) domain $T_{U}$, which contains our discrete domain $\Omega_{h}$, in the following way:

$$
T_{U}=\left\{(x, y) \in \mathbb{R}^{2}: 0 \leq x \leq 1,0 \leq y \leq x\right\} .
$$


Although $T_{U}$ agrees with $\hat{T}$, we use a different notation for both triangles for the sake of clarity, since $\hat{T}$ plays the standard role of the reference element in interpolation error estimates and $T_{U}$ is the domain where the extension $u^{E}$ of $u$ will be studied.

Lemma 2.6. If $1<\alpha<3$, there exists a constant $C$, which depends only on $\alpha$, such that

$$
\left\|u^{E}\right\|_{W^{2, p}\left(T_{U}\right)} \leq C\left\|u^{E}\right\|_{H_{\alpha}^{2}\left(T_{U}\right)}
$$

for all $1 \leq p<\frac{4}{\alpha+1}$.

Proof. The proof follows by using (2.10), with $s=\frac{\alpha-1}{2}<1$, for $u^{E}$ and its derivatives.

Remark 2.2. Conditions (1), (2) and (Ha), together with the fact that $\alpha>1$, imply that there exists only one triangle $T$ in the mesh such that $(0,0) \in T$. Moreover, its vertices are necessarily of the form $(0,0),\left(0, h_{1}\right)$, and $\left(x_{1}, x_{1}^{\alpha}\right)$. Furthermore, if $h_{T} \rightarrow 0$, the angle $\theta_{0}$ placed at $(0,0)$ tends to zero since $\frac{x_{1}^{\alpha}}{x_{1}}=x_{1}^{\alpha-1} \leq h_{T}^{\alpha-1} \rightarrow 0$, and, hence, condition (1) implies that for $h_{T}$ small enough $\theta_{0}$ is in fact the minimum interior angle.

Now we can prove the following "global" version of the interpolation error estimates,

Theorem 2.2. There exists a constant $C$ depending only on $\theta_{M}, \sigma$ and $\alpha$ such that

$$
\left\|\nabla\left(u^{E}-\Pi u^{E}\right)\right\|_{L^{2}\left(\Omega_{h}\right)} \leq C h\left|u^{E}\right|_{H_{\alpha}^{2}\left(\Omega_{h}\right)}
$$

and

$$
\left\|u^{E}-\Pi u^{E}\right\|_{L^{2}\left(\Omega_{h}\right)} \leq C h^{2}\left|u^{E}\right|_{H_{\alpha}^{2}\left(\Omega_{h}\right)},
$$

where $\Pi u^{E} \in V_{h}$ denotes the piecewise linear Lagrange interpolation of $u^{E}$ and $\left|u^{E}\right|_{H_{\alpha}^{2}\left(\Omega_{h}\right)}$ denotes the usual semi-norm on $H_{\alpha}^{2}$,

Proof. We will only sketch the proof because it is standard (see [15]). For (2.18) we write

$$
\left\|\nabla\left(u^{E}-\Pi u^{E}\right)\right\|_{L^{2}\left(\Omega_{h}\right)}^{2}=\left\|\nabla\left(u^{E}-\Pi u^{E}\right)\right\|_{L^{2}\left(T_{1}\right)}^{2}+\sum_{T \in \mathcal{T}_{h}, T \neq T_{1}}\left\|\nabla\left(u^{E}-\Pi u^{E}\right)\right\|_{L^{2}(T)}^{2} .
$$

We observe that, in view of Remark 2.2 , the triangle $T_{1}$ defined in (Ha) is the unique triangle which contains $(0,0)$. The first term can be bounded by using condition (1), Theorem 2.1 with $s=\frac{\alpha-1}{2}<1$ (recall that $\alpha<3$ ), and noticing that Lemma 2.6 gives the necessary regularity for $u^{E}$ (use embedding results on $T_{U}$ ). Finally, condition (2) allows us to replace $h_{T_{1}}$ by $h$. The second term can be handled using error estimates for Lagrange interpolation for classical unweighted Sobolev spaces under the maximal angle condition (see, for example, [6]), together with condition (3). Indeed, since $(0,0)$ is not in $T$, we have

$$
\left\|\nabla\left(u^{E}-\Pi u^{E}\right)\right\|_{L^{2}(T)} \leq C h_{T}\left|u^{E}\right|_{H^{2}(T)} \leq C h \inf _{T} x^{\gamma}\left|u^{E}\right|_{H^{2}(T)} \leq C h\left|u^{E}\right|_{H_{\alpha}^{2}(T)} .
$$

The estimate (2.19) is handled in the same way.

We finish this section by recalling the following estimate that will be useful later on. 
Lemma 2.7. If $1<\alpha<3$, then there exists a constant $C$, which depends only on $\alpha, \theta_{M}$ and $\sigma$, such that

$$
\left\|\nabla u^{E}\right\|_{L^{2}\left(\Omega_{h} \backslash \Omega\right)} \leq C h \sqrt{\log (1 / h)}\|u\|_{H^{2}(\Omega)} .
$$

Proof. See Lemma 4.1 in 2 .

\section{3. $L^{2}$ ERRor estimates in $\Omega$ When $f \equiv 0$ OUtside $\Omega$}

In this section we obtain error estimates in $L^{2}(\Omega)$ of quasi-optimal order (i.e., optimal up to a logarithmic factor) with respect to the number of nodes using appropriate graded meshes, when $f$ vanishes outside $\Omega$.

The following error estimate in $H^{1}(\Omega)$ for the finite element approximation of the Poisson problem (1.2) with $f \equiv 0$ outside $\Omega$ was obtained in [2].

Theorem 3.1. Let $u$ be the solution of (1.2) and $u_{h} \in V_{h}$ be the solution of (1.4). Assume that $1<\alpha<3$ and that $f \in L^{2}(\Omega)$ is extended by zero outside $\Omega$.

If the family of meshes satisfies (1), (2), (3) and (Ha), then there exists a constant $C$ depending only on $\alpha, \theta_{M}$ and $\sigma$ such that

$$
\left\|u-u_{h}\right\|_{H^{1}(\Omega)} \leq\left\|u^{E}-u_{h}\right\|_{H^{1}\left(\Omega_{h}\right)} \leq C h \sqrt{\log (1 / h)}\|f\|_{L^{2}(\Omega)} .
$$

Our next goal is to obtain error estimates in the $L^{2}$ norm. In order to use the Aubin-Nitsche duality arguments we introduce the following auxiliary problem: Let $\Phi \in H^{1}(\Omega)$ be the solution of

$$
\left\{\begin{aligned}
-\Delta \Phi & =u-u_{h}, \quad \text { in } \Omega, \\
\frac{\partial \Phi}{\partial \nu} & =0, \quad \text { on } \Gamma_{1} \cup \Gamma_{3}, \\
\Phi & =0, \quad \text { on } \Gamma_{2},
\end{aligned}\right.
$$

where $\nu$ denotes the outside normal. Applying the a priori estimate (1.3) to $\Phi$, we have that $\Phi \in H^{2}(\Omega)$ and that there exists a constant $C$ such that

$$
\|\Phi\|_{H^{2}(\Omega)} \leq C\left\|u-u_{h}\right\|_{L^{2}(\Omega)} .
$$

Moreover, solutions of (3.20) can be extended to $\mathbb{R}^{2}$. Indeed, the analogue of (1.5) applied to $\Phi$ shows that there exists a function $\Phi^{E} \in H_{\alpha}^{2}\left(\mathbb{R}^{2}\right)$ such that $\left.\Phi^{E}\right|_{\Omega}=\Phi$ and

$$
\left\|\Phi^{E}\right\|_{H_{\alpha}^{2}\left(\mathbb{R}^{2}\right)} \leq C\|\Phi\|_{H^{2}(\Omega)} .
$$

On the other hand, applying Lemma 2.6 to $\Phi^{E}$, we get

$$
\left\|\Phi^{E}\right\|_{W^{2, p}\left(T_{U}\right)} \leq C\left\|\Phi^{E}\right\|_{H_{\alpha}^{2}\left(T_{U}\right)}
$$

for $1 \leq p<\frac{4}{\alpha+1}$.

Theorem 3.2. Let $u$ be the solution of (1.2) and $u_{h}$ be the solution of (1.4). Assume that $1<\alpha<3$ and that $f \in L^{2}(\Omega)$ is extended by zero outside $\Omega$. Then,

$$
\left\|u-u_{h}\right\|_{L^{2}(\Omega)} \leq C h^{2} \log (1 / h)\|f\|_{L^{2}(\Omega)} .
$$

Proof. Let $e=u-u_{h}$ and $\Phi$ be the solution of (3.20). We have that

$$
\int_{\Omega} e^{2}=\int_{\Omega}(-\Delta \Phi) e=\int_{\Omega} \nabla \Phi \nabla e=\int_{\Omega} \nabla(\Phi-\Pi \Phi) \nabla e+\int_{\Omega} \nabla(\Pi \Phi) \nabla e .
$$


From (1.2) and (1.4) we get

$$
\int_{\Omega} \nabla e \nabla v=\int_{\Omega_{h} \backslash \Omega} \nabla u_{h} \nabla v \quad \forall v \in V_{h} .
$$

Hence,

$$
\int_{\Omega} e^{2}=\int_{\Omega} \nabla(\Phi-\Pi \Phi) \nabla e+\int_{\Omega_{h} \backslash \Omega} \nabla u_{h} \nabla\left(\Pi \Phi^{E}\right) .
$$

From Theorem 2.2 applied to $\Phi^{E}$ and (3.22), we have that

$\|\nabla(\Phi-\Pi \Phi)\|_{L^{2}(\Omega)} \leq\left\|\nabla\left(\Phi^{E}-\Pi \Phi^{E}\right)\right\|_{L^{2}\left(\Omega_{h}\right)} \leq C h\left\|\Phi^{E}\right\|_{H_{\alpha}^{2}\left(\Omega_{h}\right)} \leq C h\|\Phi\|_{H^{2}(\Omega)}$.

Then, the first term of (3.25) can be bounded by means of Theorem 3.1 and (3.21). Indeed,

$$
\int_{\Omega} \nabla(\Phi-\Pi \Phi) \nabla e \leq C h^{2} \log (1 / h)\|f\|_{L^{2}(\Omega)}\|e\|_{L^{2}(\Omega)} .
$$

For the second term in (3.25) we have that

$$
\int_{\Omega_{h} \backslash \Omega} \nabla u_{h} \nabla\left(\Pi \Phi^{E}\right)=\int_{\Omega_{h} \backslash \Omega} \nabla\left(u_{h}-u^{E}\right) \nabla\left(\Pi \Phi^{E}\right)+\int_{\Omega_{h} \backslash \Omega} \nabla u^{E} \nabla\left(\Pi \Phi^{E}\right) .
$$

The first term can be bounded using Theorem 3.1 by

$$
\begin{aligned}
\int_{\Omega_{h} \backslash \Omega} & \nabla\left(u_{h}-u^{E}\right) \nabla\left(\Pi \Phi^{E}\right) \\
\leq & C h \sqrt{\log (1 / h)}\|f\|_{L^{2}(\Omega)}\left\{\left\|\nabla\left(\Pi \Phi^{E}-\Phi^{E}\right)\right\|_{L^{2}\left(\Omega_{h} \backslash \Omega\right)}+\left\|\nabla \Phi^{E}\right\|_{L^{2}\left(\Omega_{h} \backslash \Omega\right)}\right\},
\end{aligned}
$$

while the second term can be bounded using Lemma 2.7 and (1.3) by

$$
\begin{aligned}
\int_{\Omega_{h} \backslash \Omega} & \nabla u^{E} \nabla\left(\Pi \Phi^{E}\right) \\
\leq & C h \sqrt{\log (1 / h)}\|f\|_{L^{2}(\Omega)}\left\|\nabla \Pi \Phi^{E}\right\|_{L^{2}\left(\Omega_{h} \backslash \Omega\right)} \\
\leq & C h \sqrt{\log (1 / h)}\|f\|_{L^{2}(\Omega)}\left\{\left\|\nabla\left(\Pi \Phi^{E}-\Phi^{E}\right)\right\|_{L^{2}\left(\Omega_{h} \backslash \Omega\right)}+\left\|\nabla \Phi^{E}\right\|_{L^{2}\left(\Omega_{h} \backslash \Omega\right)}\right\} .
\end{aligned}
$$

Therefore, from (3.27) we get

$$
\begin{aligned}
\int_{\Omega_{h} \backslash \Omega} & \nabla u_{h} \nabla\left(\Pi \Phi^{E}\right) \\
\leq & C h \sqrt{\log (1 / h)}\|f\|_{L^{2}(\Omega)}\left\{\left\|\nabla\left(\Pi \Phi^{E}-\Phi^{E}\right)\right\|_{L^{2}\left(\Omega_{h} \backslash \Omega\right)}+\left\|\nabla \Phi^{E}\right\|_{L^{2}\left(\Omega_{h} \backslash \Omega\right)}\right\},
\end{aligned}
$$

and using (3.26), Lemma 2.7 applied to $\Phi^{E}$, and (3.21), we obtain

$$
\int_{\Omega_{h} \backslash \Omega} \nabla u_{h} \nabla\left(\Pi \Phi^{E}\right) \leq C h^{2} \log (1 / h)\|f\|_{L^{2}(\Omega)}\|e\|_{L^{2}(\Omega)},
$$

and the theorem follows.

Remark 3.1. Whether or not the logarithmic factor $\log h$ in Theorem 3.1 and Theorem 3.2 can be removed is an open problem, and it is not easy to obtain information about either possibility from numerical experiments. 


\section{Error estimates in the CASE in Which $f \not \equiv 0$ OUtside $\Omega$}

In this section we will obtain error estimates in $H^{1}$ norm and $L^{2}$ norm when $f$ does not necessarily vanish outside $\Omega$. This kind of estimate can be useful in several situations. For example, even for simple sources such as $f \equiv 1$ in $\Omega$, the term $\int_{\Omega_{h}} \chi_{\Omega} v$ in (1.4) is usually replaced by $\int_{\Omega_{h}} 1 v$ (i.e. as if $f$ were defined as 1 over $\Omega_{h}$ ) in numerical computations when any standard quadrature rule is applied. In general, the contribution of the terms such as $\int_{\Omega_{h} \backslash \Omega} \tilde{f} v_{h}$, with $\tilde{f}$ being a certain approximation of $f$ defined on $\Omega_{h}$, may be useful in order to evaluate the effect of numerical integration. On the other hand, in eigenvalue approximations the usual approach (see [9]) is based on the convergence of appropriate operators $T_{h}$ to the limit operator $T$, with $T$ being the inverse of the Laplacian. Since $\Omega_{h} \neq \Omega$, the operators $T_{h}$ are mesh dependent and the analysis leads to the study of problems such as (1.4) with $f$ not necessarily equal to zero outside $\Omega$ [17].

In order to analyze the contribution of the consistency term arising from the integral $\int_{\Omega_{h} \backslash \Omega} f v_{h}$ in equation (1.4), we will need, in addition to assumptions (1), (2), (3) and (Ha), the following hypothesis about the mesh:

(Hb) For each triangle $T_{j}$ with vertices $P_{j-1}, P_{j}$, and $R_{j}$, and for $h_{j}$ small enough, the triangle $\tilde{T}_{j}$ with vertices $\frac{P_{j-1}+R_{j}}{2}, \frac{P_{j}+R_{j}}{2}$, and $R_{j}$ (see Figure $\underline{3}$ ) does not intersect $\Omega_{h}^{j}$.

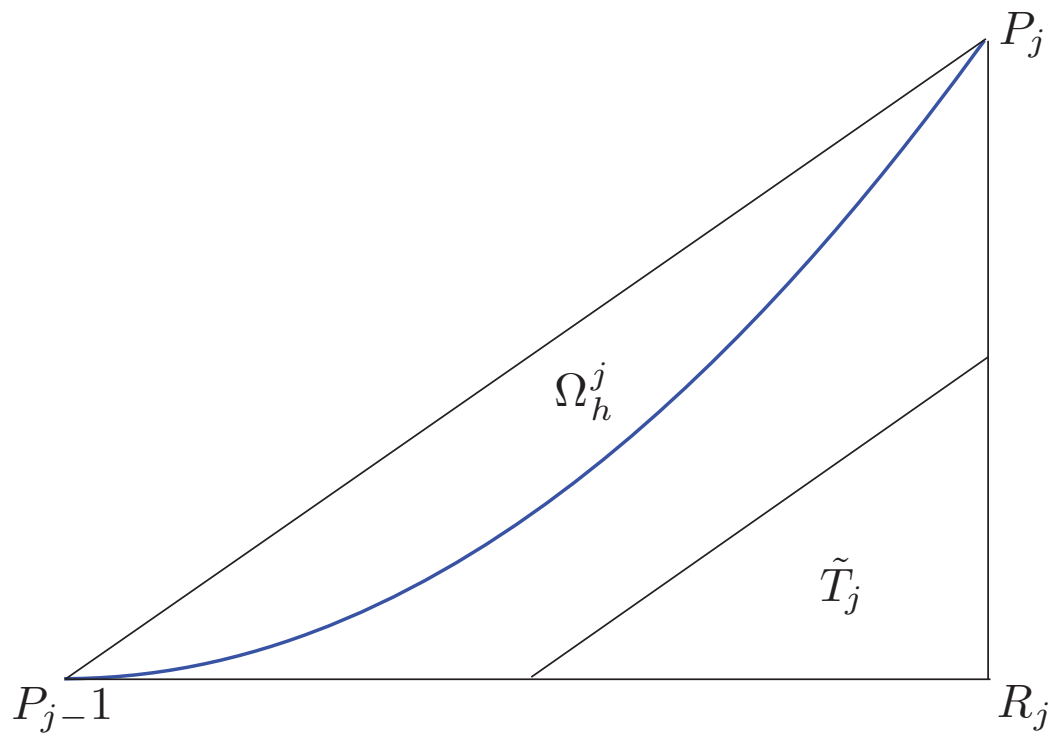

FiguRE 3

Remark 4.1. It can easily be deduced that ( $\mathrm{Hb}$ ) holds for meshes with only regular elements and for domains with smooth boundaries. Meshes for the domains under consideration in this paper involve necessarily anisotropic elements (consider, for instance, any element with a vertex at $(0,0)$ ), and this kind of element may fail to verify condition $(\mathrm{Hb})$. In fact, an easy example is given by taking $\alpha=5$ and the 
triangle $T$ defined by the vertices $(0,0),(h, 0)$, and $\left(h, h^{5}\right)$. In Section 6 we will show that meshes verifying conditions (1), (2), (3),(Ha) and ( $\mathrm{Hb})$ can be constructed (we recall that $1<\alpha<3)$.

In what follows we will assume that the family of triangulations under consideration verifies (1), (2), (3), (Ha) and (Hb).

Our first goal is to obtain the $H^{1}$ error estimates for the solutions of (1.2) and (1.4). In order to do that we will use the following result (let us recall that $\Omega_{h}$ is not uniformly Lipschitz in $h$ ):

Lemma 4.1. For any $v_{h} \in V_{h}$ there exists a constant $C$ such that

$$
\left\|v_{h}\right\|_{L^{p}\left(\Omega_{h}\right)} \leq C\left\|v_{h}\right\|_{H^{1}\left(\Omega_{h}\right)}
$$

for $1 \leq p \leq \frac{2(\alpha+1)}{\alpha-1}$.

Proof. Since $\Omega \subset \Omega_{h}$ and $v_{h} \in V_{h}$, we see that $\left.v_{h}\right|_{\Omega} \in H^{1}(\Omega)$, and then, using the imbedding result for cusps given in Theorem 5.35 of [4] (with $\nu=\alpha-1$ ), we get

$$
\left\|v_{h}\right\|_{L^{p}(\Omega)} \leq C\left\|v_{h}\right\|_{H^{1}(\Omega)}
$$

for $1 \leq p \leq \frac{2(\alpha+1)}{\alpha-1}$. We now need to show that $v_{h}$ can also be bounded on $\Omega_{h} \backslash \Omega$. More precisely, since $v_{h}$ is a piecewise linear function, we claim that

$$
\left\|v_{h}\right\|_{L^{p}\left(\Omega_{h}\right)} \leq C\left\|v_{h}\right\|_{L^{p}(\Omega)},
$$

from which we can easily obtain the desired result. Inequalities such as (4.28) for Lipschitz domains have been obtained in different works (see, for example, [16]).

Let us introduce the notation $M^{j}=T_{j} \backslash \Omega_{h}^{j}$ (i.e. $M^{j}$ stands for $\Omega \cap T_{j}$ ). All we need is to show that the local estimates

$$
\left\|v_{h}\right\|_{L^{p}\left(T_{j}\right)} \leq C\left\|v_{h}\right\|_{L^{p}\left(M^{j}\right)}
$$

hold with $C$ depending only on $\alpha$. From $(\mathrm{Hb})$ we have that $\tilde{T}_{j} \cap \Omega_{h}^{j}=\emptyset$, so $\tilde{T}_{j} \subset M^{j} \subset T_{j}$. On the other hand, calling $\hat{T}$ and $\hat{T}_{\frac{1}{2}}$ the triangles of vertices $\{(0,0),(1,0),(0,1)\}$ and $\left\{(0,0),\left(\frac{1}{2}, 0\right),\left(0, \frac{1}{2}\right)\right\}$ respectively, we have that there exists an affine mapping $\hat{F}$ such that $\hat{F}(\hat{T})=T_{j}$ and $\hat{F}\left(\hat{T}_{\frac{1}{2}}\right)=\tilde{T}_{j}$. Now, since the space of linear functions has finite dimension, we have

$$
\|\hat{v}\|_{L^{p}(\hat{T})} \leq C\|\hat{v}\|_{L^{p}\left(\hat{T}_{\frac{1}{2}}\right)}
$$

for any linear function $\hat{v}$ (the constant $C$ depends only on $\hat{T}$ and $\hat{T}_{\frac{1}{2}}$ ). Changing variables we get

$$
\left\|v_{h}\right\|_{L^{p}\left(T_{j}\right)} \leq C\left\|v_{h}\right\|_{L^{p}\left(\tilde{T}_{j}\right)},
$$

from which (4.29) follows, since $\tilde{T}_{j} \subset M^{j}$.

Remark 4.2. Note that, since functions in $V_{h}$ vanish at $\Gamma_{2}$, the previous lemma implies, together with Poincaré inequality, that

$$
\left\|v_{h}\right\|_{L^{p}\left(\Omega_{h}\right)} \leq C\left|v_{h}\right|_{H^{1}\left(\Omega_{h}\right)}
$$

for $1 \leq p \leq \frac{2(\alpha+1)}{\alpha-1}$.

Theorem 4.1 below is a generalization of Theorem 3.1 and essentially says that the contribution to the error of the consistency type term due to fact that $f \not \equiv 0$ outside $\Omega$ is at most $C h^{\frac{2}{\alpha+1}}\|f\|_{L^{2}\left(\Omega_{h} \backslash \Omega\right)}$. 
Theorem 4.1. Let $u$ be the solution of (1.2) and $u_{h}$ be the solution of (1.4). If we assume that $\alpha<3$ and $f \in L^{2}\left(\mathbb{R}^{2}\right)$, then there exists a positive constant $C$, depending only on $\alpha, \theta_{M}$, and $\sigma$, such that

$$
\left\|u-u_{h}\right\|_{H^{1}(\Omega)} \leq\left\|u^{E}-u_{h}\right\|_{H^{1}\left(\Omega_{h}\right)} \leq C h \sqrt{\log 1 / h}\|f\|_{L^{2}(\Omega)}+C h^{\frac{2}{\alpha+1}}\|f\|_{L^{2}\left(\Omega_{h} \backslash \Omega\right)} .
$$

Proof. Since $\Omega \subset \Omega_{h}$, by Poincaré inequality and (1.3) we observe that it is enough to prove that

$$
\left|u^{E}-u_{h}\right|_{H^{1}\left(\Omega_{h}\right)} \leq C h \sqrt{\log 1 / h}\|u\|_{H^{2}(\Omega)}+C h^{\frac{2}{\alpha+1}}\|f\|_{L^{2}\left(\Omega_{h} \backslash \Omega\right)} .
$$

Now,

$$
\left|u^{E}-u_{h}\right|_{H^{1}\left(\Omega_{h}\right)}^{2}=\int_{\Omega_{h}} \nabla\left(u^{E}-u_{h}\right) \cdot \nabla\left(u^{E}-\Pi u^{E}\right)+\int_{\Omega_{h}} \nabla\left(u^{E}-u_{h}\right) \cdot \nabla\left(\Pi u^{E}-u_{h}\right),
$$

but we know from (1.5) and (2.18) that

$$
\left|u^{E}-\Pi u^{E}\right|_{H^{1}\left(\Omega_{h}\right)} \leq C h\left\|u^{E}\right\|_{H_{\alpha}^{2}\left(\Omega_{h}\right)} \leq C h\|u\|_{H^{2}(\Omega)} .
$$

Thus, for the first term in (4.32), by Young's inequality, we have

$$
\int_{\Omega_{h}} \nabla\left(u^{E}-u_{h}\right) \cdot \nabla\left(u^{E}-\Pi u^{E}\right) \leq \varepsilon\left|u^{E}-u_{h}\right|_{H^{1}\left(\Omega_{h}\right)}^{2}+C_{\varepsilon} h^{2}\|u\|_{H^{2}(\Omega)}^{2},
$$

with $\varepsilon$ to be chosen below.

For the second term of (4.32) we proceed as follows. Let us introduce the notation $w_{h}:=\Pi u^{E}-u_{h}$. From (1.2) and (1.4) we have

$$
\begin{aligned}
\int_{\Omega_{h}} \nabla\left(u^{E}-u_{h}\right) \cdot \nabla w_{h} & =\int_{\Omega_{h}} \nabla u^{E} \cdot \nabla w_{h}-\int_{\Omega_{h}} \nabla u_{h} \cdot \nabla w_{h} \\
& =\int_{\Omega_{h} \backslash \Omega} \nabla u^{E} \cdot \nabla w_{h}-\int_{\Omega_{h} \backslash \Omega} f w_{h} .
\end{aligned}
$$

From Lemma 2.7 using Young's inequality again we obtain

$$
\left|\int_{\Omega_{h} \backslash \Omega} \nabla u^{E} \cdot \nabla w_{h}\right| \leq C_{\varepsilon} h^{2} \log (1 / h)\|u\|_{H^{2}(\Omega)}^{2}+\varepsilon\left|w_{h}\right|_{H^{1}\left(\Omega_{h}\right)}^{2},
$$

while for the second term in (4.34), if we take $\frac{1}{p}+\frac{1}{q}=1$ as

$$
q=2 \frac{\alpha+1}{\alpha+3}<2, \quad p=2 \frac{\alpha+1}{\alpha-1}
$$

we can write

$$
\left|\int_{\Omega_{h} \backslash \Omega} f w_{h}\right| \leq\left(\int_{\Omega_{h} \backslash \Omega} f^{q}\right)^{\frac{1}{q}}\left(\int_{\Omega_{h} \backslash \Omega} w_{h}^{p}\right)^{\frac{1}{p}} \leq\left(\int_{\Omega_{h} \backslash \Omega} f^{q}\right)^{\frac{1}{q}}\left(\int_{\Omega_{h}} w_{h}^{p}\right)^{\frac{1}{p}} .
$$

Again applying Hölder's inequality and Lemma 4.1 to the limit case $p=2 \frac{\alpha+1}{\alpha-1}$ $\left(w_{h} \in V_{h}\right.$; see also (4.30) $)$, we get

$$
\left|\int_{\Omega_{h} \backslash \Omega} f w_{h}\right| \leq C\left|\Omega_{h} \backslash \Omega\right|^{\frac{2-q}{2 q}}\|f\|_{L^{2}\left(\Omega_{h} \backslash \Omega\right)}\left|w_{h}\right|_{H^{1}\left(\Omega_{h}\right)},
$$

and by Young's inequality, Lemma 2.3, and replacing $q=2 \frac{\alpha+1}{\alpha+3}$, we obtain

$$
\left|\int_{\Omega_{h} \backslash \Omega} f w_{h}\right| \leq C_{\varepsilon} h^{\frac{4}{\alpha+1}}\|f\|_{L^{2}\left(\Omega_{h} \backslash \Omega\right)}^{2}+\varepsilon\left|w_{h}\right|_{H^{1}\left(\Omega_{h}\right)}^{2} .
$$


This inequality, together with (4.34) and (4.35), gives

$$
\begin{aligned}
\left|\int_{\Omega_{h}} \nabla\left(u^{E}-u_{h}\right) \cdot \nabla w_{h}\right| \leq & C_{\varepsilon} h^{2} \log (1 / h)\|u\|_{H^{2}(\Omega)}^{2} \\
& +C_{\varepsilon} h^{\frac{4}{\alpha+1}}\|f\|_{L^{2}\left(\Omega_{h} \backslash \Omega\right)}^{2}+2 \varepsilon\left|w_{h}\right|_{H^{1}\left(\Omega_{h}\right)}^{2} .
\end{aligned}
$$

By (2.18)

$$
\begin{aligned}
\left|w_{h}\right|_{H^{1}\left(\Omega_{h}\right)}^{2} & \leq 2\left(\left|\Pi u^{E}-u^{E}\right|_{H^{1}\left(\Omega_{h}\right)}^{2}+\left|u^{E}-u_{h}\right|_{H^{1}\left(\Omega_{h}\right)}^{2}\right) \\
& \leq C h^{2}\|u\|_{H^{2}(\Omega)}^{2}+2\left|u^{E}-u_{h}\right|_{H^{1}\left(\Omega_{h}\right)}^{2},
\end{aligned}
$$

and replacing (4.37) in (4.36) we get ( $C_{\varepsilon}$ may change from line to line)

$$
\begin{aligned}
\left|\int_{\Omega_{h}} \nabla\left(u^{E}-u_{h}\right) \cdot \nabla w_{h}\right| & \leq C_{\varepsilon} h^{2} \log (1 / h)\|u\|_{H^{2}(\Omega)}^{2}+C_{\varepsilon} h^{\frac{4}{\alpha+1}}\|f\|_{L^{2}\left(\Omega_{h} \backslash \Omega\right)}^{2} \\
& +4 \varepsilon\left|\tilde{u}-u_{h}\right|_{H^{1}\left(\Omega_{h}\right)}^{2} .
\end{aligned}
$$

Finally, taking $\varepsilon$ small enough, by (4.32), (4.33) and (4.38) we obtain (4.31), and the theorem follows.

Our next goal is to obtain error estimates in $L^{2}(\Omega)$.

Theorem 4.2. Let $u$ be the solution of (1.2) and $u_{h}$ be the solution of (1.4). Assume $\alpha<3$ and $f \in L^{2}\left(\mathbb{R}^{2}\right)$. Then

$$
\left\|u-u_{h}\right\|_{L^{2}(\Omega)} \leq C h^{2} \log (1 / h)\|f\|_{L^{2}(\Omega)}+C h\|f\|_{L^{2}\left(\Omega_{h} \backslash \Omega\right)} .
$$

Proof. Let $e=u-u_{h}$ and $\Phi$ be the solution of (3.20). Then

$$
\int_{\Omega} e^{2}=\int_{\Omega}(-\Delta \Phi) e=\int_{\Omega} \nabla \Phi \nabla e=\int_{\Omega} \nabla(\Phi-\Pi \Phi) \nabla e+\int_{\Omega} \nabla(\Pi \Phi) \nabla e .
$$

From (1.2) and (1.4) we get

$$
\int_{\Omega} \nabla e \nabla v=\int_{\Omega_{h} \backslash \Omega} \nabla u_{h} \nabla v-\int_{\Omega_{h} \backslash \Omega} f v \quad \forall v \in V_{h},
$$

hence,

$$
\int_{\Omega} e^{2}=\int_{\Omega} \nabla(\Phi-\Pi \Phi) \nabla e+\int_{\Omega_{h} \backslash \Omega} \nabla u_{h} \nabla\left(\Pi \Phi^{E}\right)-\int_{\Omega_{h} \backslash \Omega} f\left(\Pi \Phi^{E}\right) .
$$

The first term of (4.39) can be bounded by means of Theorem 4.1, (3.26) and the a priori estimates (3.21). Indeed,

$$
\int_{\Omega} \nabla(\Phi-\Pi \Phi) \nabla e \leq C h\left\{h \sqrt{\log (1 / h)}\|f\|_{L^{2}(\Omega)}+h^{\frac{2}{\alpha+1}}\|f\|_{L^{2}\left(\Omega_{h} \backslash \Omega\right)}\right\}\|e\|_{L^{2}(\Omega)} .
$$

For the second term in (4.39), using Lemma 2.7 and Theorem 4.1 we know that

$$
\begin{aligned}
\int_{\Omega_{h} \backslash \Omega} & \nabla u_{h} \nabla\left(\Pi \Phi^{E}\right) \\
= & \int_{\Omega_{h} \backslash \Omega} \nabla\left(u_{h}-u^{E}\right) \nabla\left(\Pi \Phi^{E}\right)+\int_{\Omega_{h} \backslash \Omega} \nabla u^{E} \nabla\left(\Pi \Phi^{E}\right) \\
\leq & C h \sqrt{\log (1 / h)}\|f\|_{L^{2}(\Omega)}\left\{\left\|\nabla\left(\Pi \Phi^{E}-\Phi^{E}\right)\right\|_{L^{2}\left(\Omega_{h} \backslash \Omega\right)}+\left\|\nabla \Phi^{E}\right\|_{L^{2}\left(\Omega_{h} \backslash \Omega\right)}\right\} .
\end{aligned}
$$


Then, using (3.26), Lemma 2.7 applied to $\Phi^{E}$, and (3.21), we get

$$
\int_{\Omega_{h} \backslash \Omega} \nabla u_{h} \nabla\left(\Pi \Phi^{E}\right) \leq C h^{2} \log (1 / h)\|f\|_{L^{2}(\Omega)}\|e\|_{L^{2}(\Omega)} .
$$

Therefore, we only have to estimate the third term in (4.39):

$$
\int_{\Omega_{h} \backslash \Omega} f\left(\Pi \Phi^{E}\right)=\int_{\Omega_{h} \backslash \Omega} f\left(\Pi \Phi^{E}-\Phi^{E}\right)+\int_{\Omega_{h} \backslash \Omega} f \Phi^{E} .
$$

Now, the $L^{2}$ interpolation error estimate given in Theorem 2.2 says that

$$
\left\|\Pi \Phi^{E}-\Phi^{E}\right\|_{L^{2}\left(\Omega_{h}\right)} \leq C h^{2}\left\|\Phi^{E}\right\|_{H_{\alpha}^{2}\left(\Omega_{h}\right)},
$$

and then, using (3.21) and (3.22) we get

$$
\int_{\Omega_{h} \backslash \Omega} f\left(\Pi \Phi^{E}-\Phi^{E}\right) \leq C h^{2}\|e\|_{L^{2}\left(\Omega_{h}\right)}\|f\|_{L^{2}\left(\Omega_{h} \backslash \Omega\right)} .
$$

Now, for the second term in (4.40) we use (3.23) and the fact that for $p>1$, functions in $W^{2, p}\left(T_{U}\right)$ are bounded, together with (3.21), 3.22) and Lemma 2.3. to obtain

$$
\begin{aligned}
\int_{\Omega_{h} \backslash \Omega} f \Phi^{E} & \leq C\left\|\Phi^{E}\right\|_{L^{\infty}\left(T_{U}\right)}\left|\Omega_{h} \backslash \Omega\right|^{\frac{1}{2}}\|f\|_{L^{2}\left(\Omega_{h} \backslash \Omega\right)} \\
& \leq C h\left\|\Phi^{E}\right\|_{H_{\alpha}^{2}\left(T_{U}\right)}\|f\|_{L^{2}\left(\Omega_{h} \backslash \Omega\right)} \leq C h\|f\|_{L^{2}\left(\Omega_{h} \backslash \Omega\right)}\|e\|_{L^{2}(\Omega)},
\end{aligned}
$$

and the theorem follows.

\section{5. $L^{2}$ ERROR ESTIMATES Between $u^{E}$ AND $u_{h}$ IN $\Omega_{h}$}

In this section we obtain $L^{2}$ error estimates between the extended function $u^{E}$ and the numerical solution $u_{h}$ in the polygonal domain $\Omega_{h}$. The results given below allow us, in particular, to obtain a precise computation of terms like $\left\|u_{h}\right\|_{L^{2}\left(\Omega_{h} \backslash \Omega\right)}$ which, for example, provides an optimal bound for the error between $\|u\|_{L^{2}(\Omega)}$ and $\left\|u_{h}\right\|_{L^{2}\left(\Omega_{h}\right)}$. On the other hand, estimates for the error between $u^{E}$ and $u_{h}$ are useful in the analysis of the error of eigenvalue problems [17.

The approach follows the lines of the previous sections, however, several extra complications arise since the dual problem is posed over the polygonal domain $\Omega_{h}$. The main result of this section is Theorem 5.1, which is more general than Theorem 4.2. However, we want to remark that Theorem 5.1 relies on Assumption 1 below, which is not necessary for the estimates in $L^{2}(\Omega)$ obtained in the previous sections.

We recall that error estimates between the extended function $u^{E}$ and the numerical solution $u_{h}$ in the $H^{1}\left(\Omega_{h}\right)$ norm have been obtained in Theorem 4.1 .

We want to use duality arguments similar to those in the previous section. For this reason we introduce the following auxiliary problem closely related to (3.20):

For any $h$ let $\Phi_{h} \in H^{1}\left(\Omega_{h}\right)$ be the solution of

$$
\left\{\begin{aligned}
-\Delta \Phi_{h} & =u^{E}-u_{h}, \quad \text { in } \Omega_{h}, \\
\frac{\partial \Phi_{h}}{\partial \nu} & =0, \quad \text { on } \Gamma_{1} \cup \Gamma_{3, h}, \\
\Phi_{h} & =0, \quad \text { on } \Gamma_{2},
\end{aligned}\right.
$$

where $\nu$ denotes the outside normal. 
A priori estimates for (5.41) in fractional and weighted Sobolev spaces are well known. Calling $\omega_{h}$ the maximum inner angle of $\Omega_{h}$, and taking

$$
\left\{\begin{array}{l}
r_{h}=1-C \alpha(\alpha-1) h^{\frac{\alpha-1}{3-\alpha}}, \quad \text { for } \quad \alpha<2, \\
r_{h}=1-C \alpha(\alpha-1) h, \quad \text { for } \quad \alpha \geq 2
\end{array}\right.
$$

for a suitable $C$, we can assume, from Lemma 2.4 that $r_{h}<\pi / \omega_{h}$. Hence, we have that $\Phi_{h} \in H^{1+r_{h}}\left(\Omega_{h}\right)$ [15] (with $r_{h}=1$ if $\Omega_{h}$ is convex) and that [15, page 388] $\Phi_{h}$ belongs to the weighted Sobolev space $H^{2, \gamma_{h}}\left(\Omega_{h}\right)$ defined by

$$
H^{2, \gamma_{h}}\left(\Omega_{h}\right)=\left\{v: \hat{r}^{\gamma_{h}} D^{\beta} v \in L^{2}\left(\Omega_{h}\right) \quad \forall \beta,|\beta| \leq 2\right\},
$$

where

$$
\hat{r}=\min _{1 \leq j \leq n} r_{j}
$$

with $r_{j}=\sqrt{\left(x-x_{j}\right)^{2}+\left(y-x_{j}^{\alpha}\right)^{2}}$ and

$$
\left\{\begin{array}{l}
\gamma_{h}=C_{\alpha} h^{\frac{\alpha-1}{3-\alpha}}, \quad \text { for } \quad \alpha<2, \\
\gamma_{h}=C_{\alpha} h \quad \text { for } \quad \alpha \geq 2
\end{array}\right.
$$

The following a priori estimates also holds:

$$
\begin{aligned}
\left\|\Phi_{h}\right\|_{H^{1+r_{h}\left(\Omega_{h}\right)}} \leq C_{h}\left\|u^{E}-u_{h}\right\|_{L^{2}\left(\Omega_{h}\right)}, \\
\left\|\Phi_{h}\right\|_{H^{2, \gamma_{h}\left(\Omega_{h}\right)}} \leq C_{h}\left\|u^{E}-u_{h}\right\|_{L^{2}\left(\Omega_{h}\right)} .
\end{aligned}
$$

For (5.45) we refer the reader to [15, while (5.46) can be found in 7, 10].

The constants $C_{h}$ in (5.45) and (5.46) may change with the number of vertices of the polygonal domain $\Omega_{h}$ (and hence with $h$ ). On the other hand, as mentioned in [16, the classical proof for (5.45) provides very poor control of the constant $C_{h}$ (see Remark 4.3.2.6 in 15). However, in 16 it is also mentioned that for Lipschitz domains $\Omega$ with piecewise $C^{2}$ boundary, the uniform boundedness of $C_{h}$ with respect to $h$ is a plausible hypothesis for reasonable triangulations, since the constants $C_{h}$ could be bounded [16, page 141] via a boundary integral formulation. As far as we know, there is not an explicit proof of this fact in the literature, even for regular domains $\Omega$.

Although our domain $\Omega$ is not Lipschitz, it has a $C^{2}$ piecewise boundary and, in view of (1.3), the a priori estimate for $\Omega$, we consider the following assumption also plausible:

Assumption 1. Our family of triangulations $\left\{\mathcal{T}_{h}\right\}$ is such that the constants $C_{h}$ in (5.45) and in (5.46) are uniformly bounded with respect to $h$. For this reason we will drop the subindex $h$ in $C_{h}$ in further references to (5.45) and (5.46).

In order to obtain $L^{2}$ error estimates using the auxiliary problem (5.41), we will need some embedding results in $\Omega_{h}$ for the solution $\Phi_{h}$. Since $\Omega_{h}$ is not uniformly Lipschitz in $h$ (in fact, $\Omega_{h} \rightarrow \Omega$, and $\Omega$ is not a Lipschitz domain), the classical embedding theorems for Lipschitz domains do not apply, neither do the general results for cusps given in [20; since $\Phi_{h}$ belongs to a weighted Sobolev space. As a consequence, we will extend $\Phi_{h}$ to some fixed Lipschitz domain in a certain weighted Sobolev space (we recall that the extension results given in 20 do not apply in our case), and then we will get proper embedding results. Therefore, we will follow the approach given in Lemma 3.1 of [2]. 
We first extend $\Phi_{h}$ from $\Omega_{h}$ to the following domain $\mathcal{D}_{h}$ (see Figure 4 ):

$$
\mathcal{D}_{h}=\left\{(x, y) \in \mathbb{R}^{2}:-x<y<g_{h}(x), 0<x<1\right\},
$$

where $g_{h}:[0,1] \rightarrow \Gamma_{3, h}$ is a parametrization of $\Gamma_{3, h}:=\bigcup_{j} \Gamma_{3, h}^{j}$ and we show that
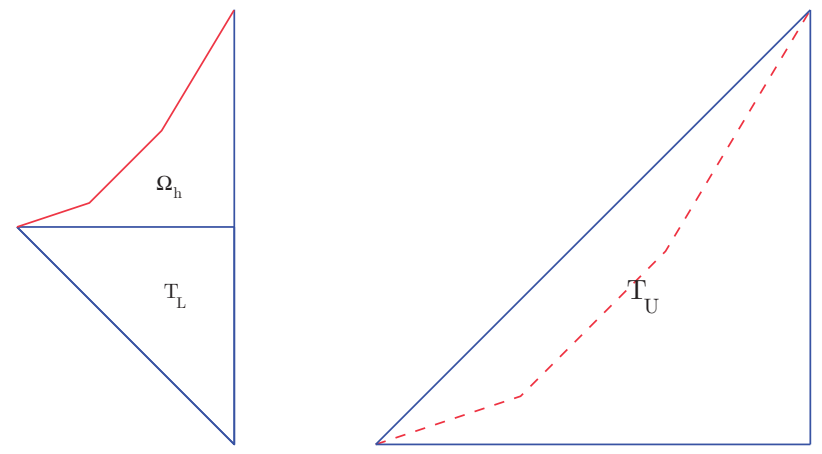

Figure 4. Left: Domain $\mathcal{D}_{h}$. Right: Triangle $T_{U}$.

the extension belongs to the space

$$
H_{\alpha+A(h)}^{2, \gamma_{h}}\left(\mathcal{D}_{h}\right)=\left\{v: r^{\frac{\alpha-1}{2}+\frac{A(h)}{2}} \rho^{\gamma_{h}} D^{\beta} v \in L^{2}\left(\mathcal{D}_{h}\right) \quad \forall \beta,|\beta| \leq 2\right\}
$$

where $A(h)=6 \gamma_{h}, r=\sqrt{x^{2}+y^{2}}$ and

$$
\rho=\min _{1 \leq j \leq n}\left\{r_{j}, d_{j}\right\}
$$

with $r_{j}=\sqrt{\left(x-x_{j}\right)^{2}+\left(y-x_{j}^{\alpha}\right)^{2}}$ and $d_{j}=\sqrt{\left(x-x_{j}\right)^{2}+\left(y+x_{j}\right)^{2}}$.

In the next lemma we find equivalent expressions for the distances involved in the weights.

Lemma 5.1. Let us denote by $d_{\Gamma_{3}}(x, \eta)$ the distance from $(x, \eta) \in \Omega$ to $\Gamma_{3}$. Then

$$
d_{\Gamma_{3}}(x, \eta) \leq x^{\alpha}-\eta \leq C d_{\Gamma_{3}}(x, \eta),
$$

with $C$ depending only on $\alpha$.

What is more, a similar discrete version of this property holds. Indeed, for any sequence $0=x_{0}<x_{1}<\cdots<x_{N}=1$, if we define $\Omega_{j}=\left\{(x, \eta): x_{j-1} \leq x \leq\right.$ $\left.x_{j}, 0 \leq \eta \leq x^{\alpha}\right\}, 1 \leq j \leq N$, then for any $(x, \eta) \in \Omega_{j}$ there exists a constant $C$ depending only on $\alpha$ such that

$$
\rho(x, \eta) \leq \min _{i=j-1, j}\left\{r_{i}(x, \eta)\right\} \leq C \rho(x, \eta),
$$

where $r_{i}(x, \eta)$ stands for the distance from $(x, \eta)$ to $\left(x_{i}, x_{i}^{\alpha}\right)$, and $\rho(x, \eta)=$ $\min _{1 \leq i \leq N}\left\{r_{i}(x, \eta)\right\}$.

Proof. It is clear that $d_{\Gamma_{3}}(x, \eta) \leq x^{\alpha}-\eta$. On the other hand, denoting by $P_{*} \in \Gamma_{3}$, $P_{*}=\left(x_{*}, x_{*}^{\alpha}\right)$ the point for which $d_{\Gamma_{3}}(x, \eta)=\left\|P_{*}-(x, \eta)\right\|$, and by $L$ the line joining the point $P_{*}$ with $\left(x, x^{\alpha}\right) \in \Gamma_{3}$, we get that $d_{L}$, the distance from $(x, \eta)$ to $L$, verifies $d_{L}(x, \eta) \leq d_{\Gamma_{3}}(x, \eta)$ (since $\left.P_{*} \in L \cap \Gamma_{3}\right)$.

Let us consider the point $Q_{*} \in L$ such that $d_{L}(x, \eta)=\left\|Q_{*}-(x, \eta)\right\|$, and let us also consider the triangle given by the points $Q_{*},(x, \eta)$ and $\left(x, x^{\alpha}\right)$. This triangle has a right angle at $Q_{*}$, and the angle $\theta$ placed at $\left(x, x^{\alpha}\right)$ is clearly bounded by 
below by some fixed $\theta_{0}>0$ depending only on $\alpha$. Now (5.48) follows because of the following inequalities:

$$
\begin{aligned}
d_{\Gamma_{3}}(x, \eta) & \geq d_{L}(x, \eta)=\left\|\left(Q_{*}-(x, \eta)\right)\right\|=\left\|\left(x, x^{\alpha}\right)-(x, \eta)\right\| \sin (\theta) \\
& \geq\left\|\left(x, x^{\alpha}\right)-(x, \eta)\right\| \sin \left(\theta_{0}\right)=\sin \left(\theta_{0}\right)\left(x^{\alpha}-\eta\right) .
\end{aligned}
$$

Let us now consider (5.49). A direct calculation shows that the function $h$ : $(0,1) \rightarrow \mathbb{R}, h(t)=(t-x)^{2}+\left(t^{\alpha}-\eta\right)^{2}$, decreases before its global minimum and increases after that. Indeed, if $x_{*}$ verifies $h^{\prime}\left(x_{*}\right)=0$, with $h^{\prime}(t)=2(t-x)+$ $2 \alpha\left(t^{\alpha}-\eta\right) t^{\alpha-1}$, hence $x_{*} \in\left[\eta^{1 / \alpha}, x\right]$ since obviously $h^{\prime}(t)<0$ for $t<\eta^{1 / \alpha}$ and $h^{\prime}>0$ for $t>x$. On the other hand, $h^{\prime \prime}(t)>0$ for $\eta^{1 / \alpha} \leq t \leq x$, which shows the existence of a unique $x_{*} \in\left[\eta^{1 / \alpha}, x\right]$ global minimum of $h$.

For $(x, \eta) \in \Omega_{j}$ and $P_{*}=\left(x_{*}, x_{*}^{\alpha}\right)$, the point for which $d_{\Gamma_{3}}(x, \eta)=\| P_{*}-$ $(x, \eta) \|=\sqrt{h\left(x_{*}\right)}$, we consider the index $l$ such that $P_{*} \in \Omega_{l}$. If $l=j$, then $\min _{j-1 \leq i \leq j}\left\{r_{i}(x, \eta)\right\}=\min _{1 \leq i \leq N}\left\{r_{i}(x, \eta)\right\}$ (since $h$ is increasing for $t>x_{*}$ and decreasing if $\left.t<x_{*}\right)$. If $l \neq j$, then, without loss of generality, we may assume $l<j$, and we write

$$
\rho(x, \eta)=\min _{1 \leq i \leq N}\left\{r_{i}(x, \eta)\right\}=\min _{l-1 \leq i \leq l}\left\{r_{i}(x, \eta)\right\} \leq \sqrt{h(x)}=x^{\alpha}-\eta \leq C d_{\Gamma_{3}}(x, \eta),
$$

where we have used the fact that $x \geq x_{l}$, the point $\left(x, x^{\alpha}\right) \in \Gamma_{3}, h$ is increasing in the range $\left[x_{l}, x\right]$, and (5.48). Now, (5.49) follows from the fact that $d_{\Gamma_{3}}(x, \eta) \leq$ $\min _{j-1 \leq i \leq j}\left\{r_{i}(x, \eta)\right\}$.

Remark 5.1. It is easy to see that for $(x, \eta) \in \Omega_{h} \subset \mathcal{D}_{h}, \rho=\hat{r}$, where $\hat{r}$ and $\rho$ are defined in (5.43) and (5.47), respectively.

We are now ready to extend $\Phi_{h}$ to $\mathcal{D}_{h}$.

Lemma 5.2. Given $v \in H^{2, \gamma_{h}}\left(\Omega_{h}\right)$ such that $\frac{\partial v}{\partial \nu}=0$ on $\Gamma_{1}$, there exists a function $\tilde{v} \in H_{\alpha+A(h)}^{2, \gamma_{h}}\left(\mathcal{D}_{h}\right)$ such that $\left.\tilde{v}\right|_{\Omega_{h}}=v$ and

$$
\|\tilde{v}\|_{H_{\alpha+A(h)}^{2, \gamma_{h}}\left(\mathcal{D}_{h}\right)} \leq C\|v\|_{H^{2, \gamma_{h}(\Omega)}},
$$

where $A(h)=6 \gamma_{h}$ and, in particular, $A(h) \rightarrow 0$ when $h \rightarrow 0$.

Proof. The proof follows the ideas given in Lemma 3.1 of [2]. We extend $v$ by reflection in the following way.

For any $(x, y) \in \mathcal{D}_{h}$ with $y \leq 0$, let us define $\eta=-x^{\alpha-1} y$. Observe that the function $(x, y) \rightarrow(x, \eta)$ maps $T_{L}$ onto $\Omega \subset \Omega_{h}$ (see Figure 4), and therefore, calling $T_{L}:=\mathcal{D}_{h} \backslash \overline{\Omega_{h}}=\{0 \leq x \leq 1,-x \leq y<0\}$, we can define

$$
\left\{\begin{array}{lll}
\tilde{v}(x, y)=v(x, y), & \text { for } \quad & (x, y) \in \Omega_{h}, \\
\tilde{v}(x, y)=v(x, \eta), & \text { for } \quad & (x, y) \in T_{L} .
\end{array}\right.
$$

We notice that for $(x, y) \in T_{L}$ we have $r=\sqrt{x^{2}+y^{2}} \sim x$, and, therefore, we can replace the weight $r^{\alpha-1+A(h)}$ by $x^{\alpha-1+A(h)}$ in our estimates.

Now, it is clear that

$$
\int_{T_{L}} \tilde{v}^{2}(x, y) x^{\alpha-1+A(h)} \rho^{2 \gamma_{h}}(x, y) d x d y \leq A+B
$$

with

$$
A=\int_{T_{L_{1}}} \tilde{v}^{2}(x, y) x^{\alpha-1+A(h)}\left[\left(x-x_{1}\right)^{2}+\left(y+x_{1}\right)^{2}\right]^{\gamma_{h}} d x d y
$$


and

$$
\begin{gathered}
B=\sum_{j=2}^{N} B_{j}, \\
B_{j}=\int_{T_{L_{j}}} \tilde{v}^{2}(x, y) x^{\alpha-1+A(h)} \min _{i=j-1, j}\left[\left(x-x_{i}\right)^{2}+\left(y+x_{i}\right)^{2}\right]^{\gamma_{h}} d x d y,
\end{gathered}
$$

where $T_{L j}=\left\{x_{j-1} \leq x \leq x_{j},-x \leq y \leq 0\right\}$ (notice that we have used the fact that $\rho \leq d_{j}=\sqrt{\left(x-x_{j}\right)^{2}+\left(y+x_{j}\right)^{2}}$, for any $\left.j\right)$.

Changing variables, and taking into account that $\alpha<3$, we get for $j>1$

$$
\begin{aligned}
& B_{j}=\int_{\Omega_{j}} v^{2}(x, \eta) x^{A(h)} \min _{i=j-1, j}\left\{\left(x-x_{i}\right)^{2}+\left(-\frac{\eta}{x^{\alpha-1}}+x_{i}\right)^{2}\right\}^{\gamma_{h}} d x d \eta \\
\leq & C \int_{\Omega_{j}} v^{2}(x, \eta) x^{A(h)-4 \gamma_{h}} \min _{i=j-1, j}\left\{\left(x-x_{j}\right)^{2}+\left(-\eta+x_{j} x^{\alpha-1}\right)^{2}\right\}^{\gamma_{h}} d x d \eta,
\end{aligned}
$$

where $\Omega_{j}=\left\{x_{j-1} \leq x \leq x_{j}, 0 \leq \eta \leq x^{\alpha}\right\}$. Similarly,

$$
A \leq C \int_{\Omega_{1}} v^{2}(x, \eta) x^{A(h)-4 \gamma_{h}}\left\{\left(x-x_{1}\right)^{2}+\left(-\eta+x_{1} x^{\alpha-1}\right)^{2}\right\}^{\gamma_{h}} d x d \eta .
$$

Since

$$
\left(-\eta+x_{j} x^{\alpha-1}\right)^{2} \leq C\left[\left(\eta-x_{j}^{\alpha}\right)^{2}+\left(x_{j}^{\alpha}-x_{j} x^{\alpha-1}\right)^{2}\right]
$$

using the mean value theorem, Lemma 2.1, the fact that $x, x_{j} \leq 1$, and $1<\alpha$, we obtain for $j>1$

$\left(x_{j}^{\alpha}-x_{j} x^{\alpha-1}\right)^{2} \leq C x_{j}^{2} x^{2(\alpha-2)}\left(x-x_{j}\right)^{2} \leq C \max \left\{x^{2(\alpha-2)}, 1\right\}\left(x-x_{j}\right)^{2} \leq x^{-2}\left(x-x_{j}\right)^{2}$, and, hence,

$$
B_{j} \leq C \int_{\Omega_{j}} v^{2}(x, \eta) x^{A(h)-6 \gamma_{h}} \min _{i=j-1, j}\left\{r_{i}(x, \eta)^{2}\right\}^{\gamma_{h}} d x d \eta,
$$

where $r_{i}(x, \eta)=\sqrt{\left(x-x_{i}\right)^{2}+\left(\eta-x_{i}^{\alpha}\right)^{2}}$. Using the fact that $A(h)=6 \gamma_{h}$ and Lemma 5.1 we get

$$
B_{j} \leq C \int_{\Omega_{j}} v^{2}(x, \eta) \rho^{2 \gamma_{h}} d x d \eta .
$$

Similarly, for $j=1$ we have that

$$
\left(x_{1}^{\alpha}-x_{1} x^{\alpha-1}\right)^{2} \leq C \max \left\{x^{2(\alpha-2)}, 1\right\}\left(x-x_{1}\right)^{2} \leq C x^{-2}\left(x-x_{1}\right)^{2} .
$$

As a consequence,

$$
A \leq C \int_{\Omega_{1}} v^{2}(x, \eta) x^{A(h)-6 \gamma_{h}}\left\{r_{1}(x, \eta)^{2}\right\}^{\gamma_{h}} d x d \eta .
$$

Let us notice that, for $(x, \eta) \in \Omega_{1}$, it is clear that $r_{1}(x, \eta)=\rho(x, \eta)$; then

$$
A \leq C \int_{\Omega_{1}} v^{2}(x, \eta) \rho(x, \eta)^{2 \gamma_{h}} d x d \eta .
$$

From the previous inequality, (5.50) and (5.53), we have

$$
\int_{T_{L}} \tilde{v}^{2}(x, y) x^{\alpha-1+A(h)} \rho^{2 \gamma_{h}}(x, y) d x d y \leq \int_{\Omega} v^{2}(x, \eta) \rho(x, \eta)^{2 \gamma_{h}} d x d \eta .
$$


Bounds for the first and second derivatives of $\tilde{v}$ follow similarly using the same ideas given in Lemma 3.1 of [2] and the estimates given above. Therefore, we have proved that $\tilde{v} \in H_{\alpha+A(h)}^{2, \gamma_{h}}\left(T_{L}\right)$ and that

$$
\|\tilde{v}\|_{H_{\alpha+A(h)}^{2, \gamma_{h}}\left(T_{L}\right)} \leq C\|v\|_{H^{2, \gamma_{h}(\Omega)}} .
$$

On the other hand, using that $\frac{\partial v}{\partial \nu}=0$ on $\Gamma_{1}$, it is easy to see that $\tilde{v} \in H_{\alpha+A(h)}^{2, \gamma_{h}}\left(\mathcal{D}_{h}\right)$, concluding the proof.

From the previous lemma we conclude that any $\tilde{\Phi}_{h} \in H^{2, \gamma_{h}}\left(\Omega_{h}\right)$ has an extension $\tilde{\Phi}_{h}^{E}$ belonging to $H_{\alpha+A(h)}^{2, \gamma_{h}}\left(\mathcal{D}_{h}\right)$. Since $\mathcal{D}_{h}$ is uniformly Lipschitz and the weights involved belong to the Muckenhoupt class $A_{2}$, we can use Chua's results [13] with the same arguments given in [2], and then $\tilde{\Phi}_{h}^{E}$ (and hence $\Phi_{h}$ ) can be extended to $\mathbb{R}^{2}$. More precisely, there exists a function $\Phi_{h}^{E}$ belonging to

$$
H_{\alpha+A(h)}^{2, \gamma_{h}}\left(\mathbb{R}^{2}\right)=\left\{v: r^{\frac{\alpha-1}{2}+\frac{A(h)}{2}} \rho^{\gamma_{h}} D^{\beta} v \in L^{2}\left(\mathbb{R}^{2}\right) \quad \forall \beta,|\beta| \leq 2\right\}
$$

such that

$$
\left\|\Phi_{h}^{E}\right\|_{H_{\alpha+A(h)}^{2, \gamma_{h}}\left(\mathbb{R}^{2}\right)} \leq C\left\|\Phi_{h}\right\|_{\hat{H}^{2, \gamma_{h}\left(\Omega_{h}\right)}} .
$$

Remark 5.2. The extension result given in (5.55) agrees with (1.5), in the sense that when $h$ goes to zero, $\gamma_{h} \rightarrow 0, A(h) \rightarrow 0$, and $\Omega_{h} \rightarrow \Omega$. We emphasize the fact that this sort of extension cannot be obtained in a direct way from the results given in [20] due to the weights involved in the space of functions.

In what follows we will make use of $\Phi_{h}^{E}$ restricted to the domain $T_{U}$ (see (2.17) and Figure 4). Let us notice that $\Omega_{h} \subset T_{U}$ for any $0<h$, and, for $(x, y) \in T_{U}$, we have that $\min _{1 \leq j \leq n} r_{j} \leq \min _{1 \leq j \leq n} d_{j}$, and $r \sim x$. Therefore, we can state the following result (see ([5.46) and Assumption 1).

Lemma 5.3. There exists an extension $\Phi_{h}^{E}$ of $\Phi_{h}$ (the solution of (5.41)) belonging to the space

$$
H_{\alpha+A(h)}^{2, \gamma_{h}}\left(T_{U}\right)=\left\{v: x^{\frac{(\alpha-1)}{2}+\frac{A(h)}{2}} \rho^{\gamma_{h}} D^{\beta} v \in L^{2}\left(T_{U}\right) \quad \forall \beta,|\beta| \leq 2\right\},
$$

where

$$
\begin{gathered}
T_{U}=\left\{(x, y) \in \mathbb{R}^{2}: 0<y<x \quad 0<x<1\right\} \\
\text { and } \rho=\min _{1 \leq j \leq n}\left\{r_{j}\right\} \text {, with } r_{j}=\sqrt{\left(x-x_{j}\right)^{2}+\left(y-x_{j}^{\alpha}\right)^{2}} \text {. Moreover, } \\
\left\|\Phi_{h}^{E}\right\|_{H_{\alpha+A(h)}^{2, \gamma_{h}}\left(T_{U}\right)} \leq\left\|\Phi_{h}\right\|_{H^{2, \gamma_{h}\left(\Omega_{h}\right)} \leq C\left\|u^{E}-u_{h}\right\|_{L^{2}\left(\Omega_{h}\right)} .}
\end{gathered}
$$

Lemma 5.4. Let $\Phi_{h}$ be the solution of (5.41) and $\Phi_{h}^{E}$ be the extension defined in Lemma 5.3. For $h$ small enough we have:

(1) $\Phi_{h}^{E} \in W^{2, p}\left(T_{U}\right)$ for $1 \leq p<\frac{4}{1+\alpha}$. Moreover,

$$
\left\|\Phi_{h}^{E}\right\|_{W^{2, p}\left(T_{U}\right)} \leq C\left\|\Phi_{h}^{E}\right\|_{H_{\alpha+A(h)}^{2, \gamma_{h}}\left(T_{U}\right)}
$$

with a constant $C$ independent of $h$.

(2) $\nabla \Phi_{h} x^{\beta} \in W^{1, s}\left(T_{U}\right)$ for $\beta>\frac{\alpha-1}{2}$, and $s=2-\epsilon$ with $\epsilon>4 \gamma_{h}$. Moreover,

$$
\left\|\nabla \Phi_{h}^{E} x^{\beta}\right\|_{W^{1, s}\left(T_{U}\right)} \leq C\left\|\Phi_{h}^{E}\right\|_{H_{\alpha+A(h)}^{2, \gamma_{h}}\left(T_{U}\right)}
$$

with a constant $C$ independent of $h$. 
(3) With $\beta$ and $s$ as in (2), we have that $\nabla \Phi_{h}^{E} x^{\beta} \in L^{s^{*}}\left(T_{U}\right)$, with $s^{*}=\frac{2 s}{2-s}=$ $\frac{2(2-\varepsilon)}{\varepsilon}$. Moreover,

$$
\left\|\nabla \Phi_{h}^{E} x^{\beta}\right\|_{L^{s^{*}\left(T_{U}\right)}} \leq \frac{C}{\varepsilon}\left\|\Phi_{h}^{E}\right\|_{H_{\alpha+A(h)}^{2, \gamma_{h}}\left(T_{U}\right)}
$$

with a constant $C$ independent of $h$.

Proof. Let us write

$$
\int_{T_{U}}|v|^{p}=\int_{T_{U}}|v|^{p} x^{\frac{p(\alpha-1+A(h))}{2}} \rho^{p \gamma_{h}} x^{-\frac{p(\alpha-1+A(h))}{2}} \rho^{-p \gamma_{h}}
$$

for some $p<\frac{4}{\alpha+1}<2$ fixed. Applying Hölder's inequality with exponent $2 / p$ and its dual exponent we obtain

$$
\int_{T_{U}}|v|^{p} \leq\left(\int_{T_{U}}|v|^{2} x^{(\alpha-1+A(h))} \rho^{2 \gamma_{h}}\right)^{\frac{p}{2}}\left(\int_{T_{U}} x^{-\frac{p}{2-p}(\alpha-1+A(h))} \rho^{-\frac{2 p}{2-p} \gamma_{h}}\right)^{\frac{2-p}{2}} .
$$

Calling $s_{h}=\frac{2 p}{2-p} \gamma_{h}$, and applying Hölder's inequality again with $\frac{1}{1-2 s_{h}}$ (its dual exponent is $\left.\frac{1}{2 s_{h}}\right)$, we have

$$
\left(\int_{T_{U}} x^{-\frac{p(\alpha-1+A(h))}{2-p}} \rho^{-\frac{2 p}{2-p} \gamma_{h}}\right)^{\frac{2-p}{2}} \leq I_{1} I_{2}
$$

where

$$
I_{1}=\left(\int_{T_{U}} x^{-(\alpha-1+A(h)) \frac{p}{(2-p)\left(1-2 s_{h}\right)}}\right)^{\frac{2-p}{2}\left(1-2 s_{h}\right)}
$$

and

$$
I_{2}=\left(\int_{T_{U}} \rho^{-\frac{1}{2}}\right)^{(2-p) s_{h}} .
$$

Now, since $1 \leq p<\frac{4}{1+\alpha}$, for $h$ small enough we can assume that $1 \leq p<\frac{4}{1+\alpha+14 \gamma_{h}}$, and using the fact that $A(h)=6 \gamma_{h}$, one can easily check that

$$
(\alpha-1+A(h)) \frac{p}{(2-p)\left(1-2 s_{h}\right)}<2,
$$

which is precisely the condition that implies

$$
I_{1} \leq C
$$

with $C=C(\alpha)$.

On the other hand, since

$$
\rho \geq d_{\Gamma_{3}},
$$

where $d_{\Gamma_{3}}$ is the distance function to $\Gamma_{3}$, and taking into account that $\int_{T_{U}} \frac{1}{d^{s}}<C$ for any $s<1$ (see for instance [15]), we get that $\int_{T_{U}} \rho^{-\frac{1}{2}} \leq C$. As a consequence, we have proved that for any function $v$ and $1 \leq p<\frac{4}{\alpha+1+14 \gamma_{h}}$,

$$
\|v\|_{L^{p}\left(T_{U}\right)} \leq C\left\|v x^{\frac{\alpha-1+A(h)}{2}} \rho^{\gamma_{h}}\right\|_{L^{2}\left(T_{U}\right)} .
$$

Thanks to Lemma 5.3, we conclude that $\Phi_{h}^{E} \in W^{2, p}\left(T_{U}\right)$, and (1) follows. 
Our next goal is to prove (2). Take $\beta>\frac{\alpha-1}{2}$; then, for $h$ small enough, we also have $\beta>\frac{(\alpha-1+A(h))}{2}$. Let $s=2-\epsilon$, with $\epsilon$ to be chosen below. Following similar arguments as those of Lemma 4.1 of [2], we have that

$$
D^{2} \Phi_{h}^{E} x^{\beta} \in L^{2-\epsilon}\left(T_{U}\right) .
$$

Indeed, since $D^{2} \Phi_{h}^{E} x^{\beta} \rho^{\gamma_{h}} \in L^{2}\left(T_{U}\right)$, we can write for fixed $\epsilon$,

$$
\int_{T_{U}}\left|D^{2} \Phi_{h}^{E}\right|^{s} x^{s \beta} \leq\left(\int_{T_{U}}\left(D^{2} \Phi_{h}^{E} x^{\beta} \rho^{\gamma_{h}}\right)^{2}\right)^{\frac{2-\epsilon}{2}}\left(\int_{T_{U}} \rho^{-\frac{2 \gamma_{h}}{\epsilon}}\right)^{\frac{\epsilon}{2}}
$$

and the last integral in the previous inequality is finite, taking for instance

$$
4 \gamma_{h}<\epsilon
$$

and using (5.61). On the other hand,

$$
\nabla \Phi_{h}^{E} x^{\beta-1} \in L^{2}\left(T_{U}\right) .
$$

In fact, from (5.56), and embedding results for the planar Lipschitz domain $T_{U}$,

$$
\nabla \Phi_{h}^{E} \in L^{p *}\left(T_{U}\right),
$$

with $p^{*}=\frac{2 p}{2-p}$ and $1 \leq p<\frac{4}{\alpha+1}$. Now, by Hölder's inequality with exponent $p^{*} / 2$ and its conjugate exponent $\frac{p}{2(p-1)}$ we get

$$
\int_{T_{U}}\left|\nabla \Phi_{h}^{E}\right|^{2} x^{2(\beta-1)} \leq\left(\int_{T_{U}}\left|\nabla \Phi_{h}^{E}\right|^{p^{*}}\right)^{\frac{2}{p^{*}}}\left(\int_{T_{U}} x^{p(\beta-1) /(p-1)}\right)^{\frac{2(p-1)}{p}} .
$$

A straightforward computation shows that the condition for the last integral to be finite is

$$
p(\beta-1) /(p-1)+2>0
$$

or, equivalently,

$$
p>\frac{2}{\beta+1} .
$$

Choose $p$ such that

$$
\frac{2}{\beta+1}<p<\frac{4}{1+\alpha}
$$

which is possible since $\beta>\frac{\alpha-1}{2} ;(2)$ follows.

The proof of (3) is now direct using the imbedding $L^{s^{*}}\left(T_{U}\right) \subset W^{1, s}\left(T_{U}\right), s^{*}=$ $\frac{2 s}{2-s}=\frac{2(2-\epsilon)}{\epsilon}$, the explicit dependence on $s$ of the constant (see the proof of Theorem 1 in [14, page 277]), and the result obtained in (2).

In fact,

$$
\left\|\nabla \Phi_{h}^{E} x^{\beta}\right\|_{L^{s^{*}}\left(T_{U}\right)} \leq \frac{C}{2-s}\left\|\nabla \Phi_{h}^{E} x^{\beta}\right\|_{W^{1, s}\left(T_{U}\right)} \leq \frac{C}{\epsilon}\left\|\Phi_{h}^{E}\right\|_{H_{\alpha}^{2, \gamma_{h}}\left(T_{U}\right)}
$$

for $s=2-\epsilon$, with $\epsilon$ verifying (5.62).

Lemma 5.5. Let $\Phi_{h}$ be the solution of (5.41). Then there exists a constant $C$ such that

and

$$
\left\|\nabla \Phi_{h}\right\|_{L^{2}\left(\Omega_{h} \backslash \Omega\right)} \leq C h \log (1 / h)\left\|u^{E}-u_{h}\right\|_{L^{2}\left(\Omega_{h}\right)}
$$

$$
\left\|\Phi_{h}\right\|_{L^{2}\left(\Omega_{h} \backslash \Omega\right)} \leq C h\left\|u^{E}-u_{h}\right\|_{L^{2}\left(\Omega_{h}\right)} .
$$


Proof. Let $\beta, s$, and $\epsilon>4 \gamma_{h}$, as in lemma 5.4. Applying Hölder's inequality with $s^{*} / 2=\frac{2-\epsilon}{\epsilon}$ and its dual exponent $q=\frac{s^{*}}{s^{*}-2}=\frac{2-\epsilon}{2-2 \epsilon}$, we have

$$
\int_{\Omega_{h} \backslash \Omega}\left|\nabla \Phi_{h}\right|^{2} \leq\left(\int_{\Omega_{h} \backslash \Omega}\left|\nabla \Phi_{h}^{E}\right|^{s^{*}} x^{\beta s^{*}}\right)^{\frac{2}{s^{*}}}\left(\int_{\Omega_{h} \backslash \Omega} x^{-2 \beta q}\right)^{\frac{1}{q}}
$$

and, therefore, from (3) in Lemma 5.4 we obtain

$$
\int_{\Omega_{h} \backslash \Omega}\left|\nabla \Phi_{h}\right|^{2} \leq \frac{C}{\epsilon^{2}}\left\|\Phi_{h}^{E}\right\|_{H_{\alpha+A(h)}^{2, \gamma_{h}}\left(T_{U}\right)}^{2}\left(\int_{\Omega_{h} \backslash \Omega} x^{-2 \beta q}\right)^{\frac{1}{q}} .
$$

From Lemma 2.2 we get (observe that the constant given in that lemma remains bounded when $q \rightarrow 1$, and in the present context $q=\frac{2-\epsilon}{2-2 \epsilon}$, while $\epsilon$ will be chosen such that $\epsilon \rightarrow 0$ when $h \rightarrow 0$ )

$$
\int_{\Omega_{h} \backslash \Omega}\left|\nabla \Phi_{h}\right|^{2} \leq \frac{C}{\epsilon^{2}}\left\|\Phi_{h}^{E}\right\|_{H_{\alpha+A(h)}^{2, \gamma_{h}}\left(T_{U}\right)}^{2} h^{\frac{2}{q}}
$$

and, since $\frac{1}{q}=\frac{2-2 \epsilon}{2-\epsilon}=1-\frac{\epsilon}{2-\epsilon}$,

$$
\int_{\Omega_{h} \backslash \Omega}\left|\nabla \Phi_{h}\right|^{2} \leq C\left(\frac{h^{1-\frac{\epsilon}{2-\epsilon}}}{\epsilon}\right)^{2}\left\|\Phi_{h}^{E}\right\|_{H_{\alpha+A(h)}^{2, \gamma_{h}}\left(T_{U}\right)}^{2} .
$$

Let us now take $\epsilon=-\frac{1}{\log (h)}$. It is clear that for $h$ small enough, $\epsilon$ verifies $\epsilon>4 \gamma_{h}$ for any choice of $\gamma_{h}$ in (5.44). Taking into account that $1-\frac{\epsilon}{2-2 \epsilon} \sim 1-\frac{1}{2} \epsilon$ for $\epsilon \rightarrow 0$, we get by standard arguments

$$
\int_{\Omega_{h} \backslash \Omega}\left|\nabla \Phi_{h}\right|^{2} \leq C h^{2} \log ^{2}(1 / h)\left\|\Phi_{h}^{E}\right\|_{H_{\alpha+A(h)}^{2, \gamma_{h}}\left(T_{U}\right)}^{2},
$$

and the first estimate of the lemma follows from Lemma 5.3 .

The estimate for $\int_{\Omega_{h} \backslash \Omega}\left|\Phi_{h}\right|^{2}$ follows immediately. Since for $p>1$ functions in $W^{2, p}\left(T_{U}\right)$ are bounded, using (5.56) we can write

$$
\begin{aligned}
\int_{\Omega_{h} \backslash \Omega}\left|\Phi_{h}\right|^{2} & \leq\left\|\Phi_{h}^{E}\right\|_{L^{\infty}\left(T_{U}\right)}^{2}\left|\Omega_{h} \backslash \Omega\right| \\
& \leq C\left\|\Phi_{h}^{E}\right\|_{W^{2, p}\left(T_{U}\right)}^{2}\left|\Omega_{h} \backslash \Omega\right| \leq C\left\|\Phi_{h}^{E}\right\|_{H_{\alpha+A(h)}^{2, \gamma_{h}\left(T_{U}\right)}}^{2}\left|\Omega_{h} \backslash \Omega\right|,
\end{aligned}
$$

and the proof concludes using Lemma 5.3 and Lemma 2.3.

Now we are ready to obtain error bounds in the $L^{2}$ norm. Mainly due to Lemma 5.5. it will not be possible (at least with the present approach; see Remark 3.1) to improve the logarithmic factor $\log h$ in the estimates. For this reason, in the intermediate computations we will replace terms such as $h^{r_{h}}$, with $r_{h}$ given by (5.42), by $C \sqrt{\log (1 / h)} h$. This can be done thanks to the bound

$$
h^{r_{h}} \leq C \sqrt{\log (1 / h)} h,
$$

that holds for $r_{h} \sim 1-C h^{s}$ with any $0<s \leq 1$, as one can easily check from the fact that

$$
\lim _{h \rightarrow 0} h^{h^{s}}=1 .
$$

Our next goal is to obtain error estimates in $L^{2}\left(\Omega_{h}\right)$. 
Theorem 5.1. Let $u$ be the solution of (1.2) and $u_{h}$ be the solution of (1.4). Assume $\alpha<3$ and $f \in L^{2}\left(\mathbb{R}^{2}\right)$. Then,

$$
\left\|u^{E}-u_{h}\right\|_{L^{2}\left(\Omega_{h}\right)} \leq C h^{2} \log (1 / h)\|f\|_{L^{2}(\Omega)}+C h\|f\|_{L^{2}\left(\Omega_{h} \backslash \Omega\right)} .
$$

Proof. Let $e=u^{E}-u_{h}$ and $\Phi_{h}$ be the solution of (5.41). We have that

$$
\int_{\Omega_{h}} e^{2}=\int_{\Omega_{h}}\left(-\Delta \Phi_{h}\right) e=\int_{\Omega_{h}} \nabla \Phi_{h} \nabla e=\int_{\Omega_{h}} \nabla\left(\Phi_{h}-\Pi \Phi_{h}\right) \nabla e+\int_{\Omega_{h}} \nabla\left(\Pi \Phi_{h}\right) \nabla e .
$$

From (1.2) and (1.4) we get

$$
\int_{\Omega_{h}} \nabla e \nabla v=\int_{\Omega_{h} \backslash \Omega} \nabla u^{E} \nabla v-\int_{\Omega_{h} \backslash \Omega} f v \quad \forall v \in V_{h} .
$$

Hence,

$$
\int_{\Omega_{h}} e^{2}=\int_{\Omega_{h}} \nabla\left(\Phi_{h}-\Pi \Phi_{h}\right) \nabla e+\int_{\Omega_{h} \backslash \Omega} \nabla u^{E} \nabla\left(\Pi \Phi_{h}\right)-\int_{\Omega_{h} \backslash \Omega} f\left(\Pi \Phi_{h}\right) .
$$

From standard estimates for the Lagrange interpolation using finite triangular elements verifying the maximal angle condition, and (5.67), we get

$$
\left\|\nabla\left(\Phi_{h}-\Pi \Phi_{h}\right)\right\|_{L^{2}\left(\Omega_{h}\right)} \leq C h^{r_{h}}\left\|\Phi_{h}\right\|_{H^{1+r_{h}\left(\Omega_{h}\right)}} \leq C h \sqrt{\log (1 / h)}\left\|\Phi_{h}\right\|_{H^{1+r_{h}\left(\Omega_{h}\right)}},
$$

which under Assumption 1 for (5.45) yields

$$
\left\|\nabla\left(\Phi_{h}-\Pi \Phi_{h}\right)\right\|_{L^{2}\left(\Omega_{h}\right)} \leq C h \sqrt{\log (1 / h)}\|e\|_{L^{2}\left(\Omega_{h}\right)} .
$$

Therefore, the first term of (5.69) can be bounded using Theorem 4.1. Indeed,

$$
\begin{aligned}
& \int_{\Omega_{h}} \nabla\left(\Phi-\Pi \Phi_{h}\right) \nabla e \\
& \quad \leq C h \sqrt{\log (1 / h)}\left\{h \sqrt{\log (1 / h)}\|f\|_{L^{2}(\Omega)}+h^{\frac{2}{\alpha+1}}\|f\|_{L^{2}\left(\Omega_{h} \backslash \Omega\right)}\right\}\|e\|_{L^{2}\left(\Omega_{h}\right)} .
\end{aligned}
$$

For the second term in (5.69), using the estimates given in Lemma 2.7 and (1.3), we know that

$$
\begin{aligned}
& \int_{\Omega_{h} \backslash \Omega} \nabla u^{E} \nabla\left(\Pi \Phi_{h}\right) \\
& \quad \leq C h \sqrt{\log (1 / h)}\|f\|_{L^{2}(\Omega)}\left\{\left\|\nabla\left(\Pi \Phi_{h}-\Phi_{h}\right)\right\|_{L^{2}\left(\Omega_{h} \backslash \Omega\right)}+\left\|\nabla \Phi_{h}\right\|_{L^{2}\left(\Omega_{h} \backslash \Omega\right)}\right\} .
\end{aligned}
$$

Using (5.70), Lemma 5.5 and Assumption 1 for (5.45), we get

$$
\int_{\Omega_{h} \backslash \Omega} \nabla u^{E} \nabla\left(\Pi \Phi_{h}\right) \leq C h^{2} \log (1 / h)\|f\|_{L^{2}(\Omega)}\|e\|_{L^{2}\left(\Omega_{h}\right)} .
$$

Therefore, we only have to estimate the third term in (5.69),

$$
\int_{\Omega_{h} \backslash \Omega} f\left(\Pi \Phi_{h}\right)=\int_{\Omega_{h} \backslash \Omega} f\left(\Pi \Phi_{h}-\Phi_{h}\right)+\int_{\Omega_{h} \backslash \Omega} f \Phi_{h} .
$$

Now, $L^{2}$ interpolation error estimates give

$$
\left\|\Pi \Phi_{h}-\Phi_{h}\right\|_{L^{2}\left(\Omega_{h}\right)} \leq C h^{1+r_{h}}\left\|\Phi_{h}\right\|_{H^{1+r_{h}\left(\Omega_{h}\right)}},
$$

and then, (5.45) with Assumption 1 and (5.67) give

$$
\int_{\Omega_{h} \backslash \Omega} f\left(\Pi \Phi_{h}-\Phi_{h}\right) \leq C h^{2} \sqrt{\log (1 / h)}\|e\|_{L^{2}\left(\Omega_{h}\right)}\|f\|_{L^{2}\left(\Omega_{h} \backslash \Omega\right)} .
$$


Now, for the second term in (5.73), by using Lemma 5.5 we have

$$
\int_{\Omega_{h} \backslash \Omega} f \Phi_{h} \leq C h\|f\|_{L^{2}\left(\Omega_{h} \backslash \Omega\right)}\|e\|_{L^{2}\left(\Omega_{h}\right)} .
$$

So, from (5.69), (5.71), (5.72) and (5.74) we get the estimate of the theorem, taking into account that the term arising from (5.71),

$$
h^{1+\frac{2}{\alpha+1}} \sqrt{\log (1 / h)}\|f\|_{L^{2}\left(\Omega_{h} \backslash \Omega\right)},
$$

is bounded, up to a multiplicative constant, by the term

$$
h\|f\|_{L^{2}\left(\Omega_{h} \backslash \Omega\right)}
$$

given in (5.74).

\section{NumericAl EXAMPLES}

Now we show that meshes verifying hypotheses (1)-(3) and (Ha)-(Hb) can be constructed by the same method given in [2].

(1) Introduce the partition of the interval $(0,1)$ given by

$$
x_{j}=\left(\frac{j}{n}\right)^{\frac{2}{3-\alpha}}, \quad 0 \leq j \leq n .
$$

(2) Take the points $\left(x_{j}, 0\right)$ in $\Gamma_{1},\left(x_{j}, x_{j}^{\alpha}\right)$ in $\Gamma_{3}$, and, for $j>1$, divide each of the vertical lines $\left\{\left(x_{j}, y\right): 0 \leq y \leq x_{j}^{\alpha}\right\}$ uniformly into subintervals such that each of them has length $\sim x_{j}-x_{j-1}$.

Figure 5 shows an example of one of these meshes.

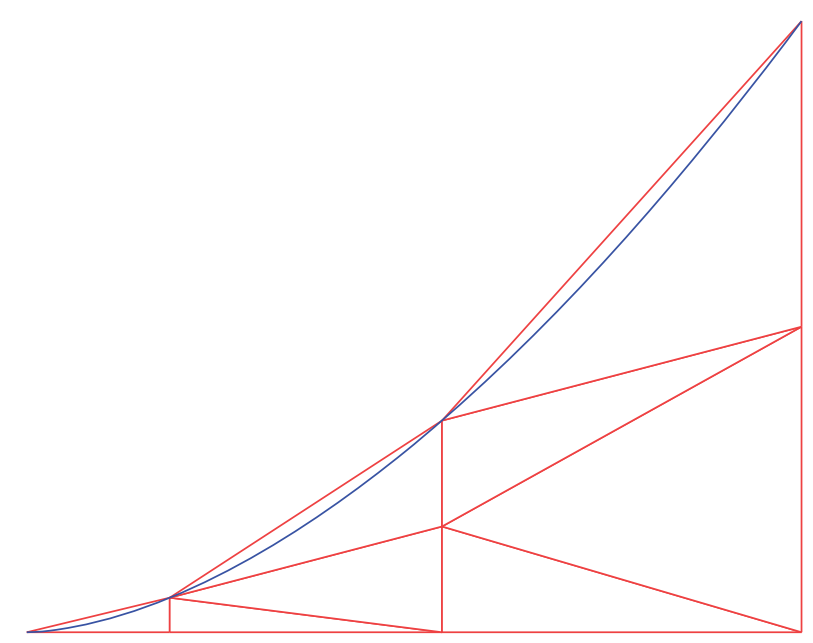

FiguRE 5. Graded mesh with $\alpha=2$ and $n=3$

We observe that is clear that the meshes constructed in this way satisfy hypotheses (1), (2), (3) and (Ha). Moreover, these meshes satisfy the additional condition $(\mathrm{Hb})$. Indeed, the first triangle $T_{1}$ has vertices $(0,0),\left(x_{1}, 0\right)$ and $\left(x_{1}, x_{1}^{\alpha}\right)$, and so 
the triangle $\tilde{T}_{1}$ has vertices $\left(\frac{x_{1}}{2}, 0\right),\left(x_{1}, 0\right)$ and $\left(x_{1}, \frac{x_{1}^{\alpha}}{2}\right)$. Then, in order to check that this triangle does not intersect $\Omega_{h}^{1}$, we analyze the function

$$
g(x)=x^{\alpha}-x_{1}^{\alpha-1}\left(x-\frac{x_{1}}{2}\right) .
$$

Hence, the hypothesis holds if we prove that $g(x)>0$ for $0 \leq x \leq x_{1}$. An easy calculation shows that $g$ is convex and has a minimum in $x^{*}=\frac{x_{1}}{\alpha^{\frac{1}{\alpha-1}}}$ and

$$
g\left(x^{*}\right)=x_{1}^{\alpha}\left(\frac{1}{2}+\frac{1}{\alpha^{\frac{\alpha}{\alpha-1}}}-\frac{1}{\alpha^{\frac{1}{\alpha-1}}}\right),
$$

which is positive for $1<\alpha<3$.

Similar arguments can be used for the rest of the triangles $T_{j}, 2 \leq j \leq n$.

If $N$ is the number of nodes in the partition $\mathcal{T}_{h}$, it can be proved that $h^{2} \sim 1 / N$ [15. page 393], 21. Therefore, if $f$ is assumed to be zero outside $\Omega$, we have the following error estimate in terms of the number of nodes:

$$
\left\|u-u_{h}\right\|_{L^{2}(\Omega)} \leq C \frac{\log N}{N}\|f\|_{L^{2}(\Omega)} .
$$

Observe that this estimate is quasi-optimal. Indeed, up to the logarithmic factor, the order with respect to the number of nodes is the same as that obtained for a smooth problem using quasi-uniform meshes.

We end this section by considering the same example presented in [2]. Here, we compare the $L^{2}$ order obtained by using uniform and graded meshes. Let us notice that we take a non-homogeneous Neumann condition, for which we know the analytical solution, and hence, the exact error. However, similar results are obtained for the same source term $f$ taking $g=0$ and by computing an estimated order of convergence from successive refinements.

Example 6.1. Consider the problem (1.1) with

$$
f(x, y)=s(s-1)\left(1+y^{2} / 2\right) x^{s-2}+x^{s}-1
$$

and

$$
g\left(t, t^{\alpha}\right)=\frac{-s \alpha t^{\alpha+s-2}\left(1+t^{2 \alpha} / 2\right)+\left(1-t^{s}\right) t^{\alpha}}{\sqrt{1+\alpha^{2} t^{2(\alpha-1)}}} .
$$

Then, the solution is

$$
u(x, y)=\left(1-x^{s}\right)\left(1+y^{2} / 2\right),
$$

and an easy calculation shows that $u \in H^{2}(\Omega)$ whenever $s>\frac{3-\alpha}{2}$.

We solve this using quasi-uniform meshes and graded meshes. Table 1 and Table 2 show the order of the error in the $L^{2}$ norm, in terms of the number of nodes and in terms of the mesh size for both kinds of meshes. Although the solution is in $H^{2}(\Omega)$, for all the values of $s$ considered, the order of convergence is not optimal when quasiuniform meshes are used. On the other hand, the optimal order of convergence is recovered by using appropriate graded meshes according to our theoretical results. 
TABLE 1. $L^{2}$ order using quasi-uniform meshes for $\alpha=2$

\begin{tabular}{|c|c|c|}
\hline value of $s$ & order in number of nodes & order in $h$ \\
\hline 0.55 & 0.769 & 1.497 \\
0.6 & 0.785 & 1.528 \\
0.65 & 0.801 & 1.561 \\
0.7 & 0.820 & 1.597 \\
0.75 & 0.842 & 1.640 \\
0.8 & 0.869 & 1.693 \\
0.85 & 0.904 & 1.761 \\
0.9 & 0.949 & 1.847 \\
0.95 & 1.001 & 1.951 \\
\hline
\end{tabular}

TABLE 2. $L^{2}$ order using graded meshes for $\alpha=2$

\begin{tabular}{|c|c|c|}
\hline value of $s$ & order in number of nodes & order in $h$ \\
\hline 0.55 & 1.090 & 2.024 \\
0.6 & 1.086 & 2.018 \\
0.65 & 1.084 & 2.013 \\
0.7 & 1.081 & 2.009 \\
0.75 & 1.080 & 2.006 \\
0.8 & 1.078 & 2.003 \\
0.85 & 1.077 & 2.001 \\
0.9 & 1.076 & 1.999 \\
0.95 & 1.076 & 1.999 \\
\hline
\end{tabular}

\section{ACKNOWLEDGMENTS}

The authors want to thank to the referees for their useful comments which helped to improve this paper. They also want to thank Ricardo G. Durán, Ariel Lombardi and Irene Drelichman for several interesting discussions.

\section{REFERENCES}

[1] G. Acosta, M. G. Armentano, R. G. Durán and A. L. Lombardi, Nonhomogeneous Neumann problem for the Poisson equation in domains with an external cusp, Journal of Mathematical Analysis and Applications 310(2), pp. 397-411, 2005. MR2022934 (2006i:35056)

[2] G. Acosta, M. G. Armentano, R. G. Durán and A. L. Lombardi, Finite element approximations in a non-Lipschitz domain, SIAM Journal on Numerical Analysis 45(1), pp. 277-295, 2007. MR2285855 (2009a:65302)

[3] G. Acosta and R. Durán, The maximum angle condition for mixed and non conforming elements: Application to the Stokes equations, SIAM J. Numer. Anal. 37, pp. 18-36, 2000. MR.1721268 (2000g:65107)

[4] R. A. Adams, Sobolev Spaces, Academic Press, New York, 1975. MR0450957 (56:9247)

[5] T. Apel, Anisotropic finite elements: Local estimates and applications, Series Advances in Numerical Mathematics, Teubner, Stuttgart, 1999. MR1716824 (2000k:65002)

[6] I. Babuska and A. K. Aziz, On the angle condition in the finite element method, SIAM J. Numer. Anal. 13, pp. 214-226, 1976. MR0455462 (56:13700)

[7] I. Babuska, R. B. Kellog, and J. Pitkaranta, Direct and inverse error estimates for finite elements with mesh refinements, Numer. Math. 33, pp. 447-471, 1979. MR553353|(81c:65054) 
[8] C. Băcută, V. Nistor, L. T. Zikatanov, Improving the rate of convergence of 'high order finite elements' on polygons and domains with cusps, Numer. Math. 100(2), pp. 165-184, 2005. MR2135780 (2006d:65130)

[9] I. Babuska and J. Osborn, Eigenvalue Problems, Handbook of Numerical Analysis, vol. II, Finite Element Methods (Part 1), 1991. MR.1115240

[10] I. Babuska and M. Suri, The P and H-P Versions of the Finite Element Method, Basic Principles and Properties, SIAM Review 36 (4), pp. 578-632, 1994. MR1306924 (96d:65184)

[11] J. J. Brannick, H. Li and L. T. Zikatanov, Uniform convergence of the multigrid $V$-cycle on graded meshes for corner singularities, Numer. Linear Algebra Appl. 15, pp. 291-306, 2008. MR2397306 (2008k:65267)

[12] S. C. Brenner, J. Cui, F. Li and L. Y. Sung, A nonconforming finite element method for a two-dimensional curl-curl and grad-div problem, Numer. Math. 109(4), pp. 509-533, 2008. MR2407321 (2009c:65294)

[13] S. K. Chua, Extension theorems on weighted Sobolev Spaces, Indiana Math. J. 41(4), pp. 1027-1076, 1992. MR.1206339 (94a:46035)

[14] L.C. Evans, Partial differential equations, Graduate Studies in Mathematics 19, American Mathematical Society, 2010. MR2597943

[15] P. Grisvard, Elliptic Problems in Nonsmooth Domains, Pitman, Boston, 1985. MR775683 (86m:35044)

[16] E. Hernández and R. Rodríguez, Finite element approximation of spectral acoustic problems on curved domains, Numer. Math. 97, pp. 131-158, 2002. MR2045461 (2005c:76063)

[17] E. Hernández and R. Rodríguez, Finite element approximation of spectral problems with Neumman boundary conditions on curved domains, Math. Comp. 72, pp. 1099-1115, 2003, MR 1972729 (2004c:65136)

[18] J. Houot and A. Munnier, On the motion and collisions of rigid bodies in an ideal fluid, Asymptot. Anal. 56, pp. 125-158, 2008. MR2394714 (2009h:76013)

[19] A. Khelif, Equations aux derivees partiellles, C.R. Acad. Sc. Paris 287, pp. 1113-1116, 1978.

[20] V. Mazya, S. Poborchi, Differentiable functions on bad domains, World Sci., Singapore, 1997. MR.1643072 (99k:46057)

[21] G. Raugel, Résolution numérique par une méthode d'éléments finis du problème Dirichlet pour le laplacien dans un polygone, C. R. Acad. Sci., Paris, Ser. A 286(18), pp. A791-A794, 1978. MR497667(81e:65060)

[22] J. A. San Martín, V. Starovoitovr and M. Tucsnak, Global weak solutions for the twodimensional motion of several rigid bodies in an incompressible viscous fluid, Arch. Ration. Mech. Anal. 161(2), pp. 113-147, 2002. MR1870954 (2002j:35259)

[23] E. Stein, Singular Integrals and Differentiablity Properties of Functions, Princeton University Press, 1970. MR0290095 (44:7280)

[24] M. Vanmaele, A. Zeníšek, The combined effect of numerical integration and approximation of the boundary in the finite element method for eigenvalue problems, Numer. Math. 71, 253-273, 1995. MR1347167 (96j:65112)

Departamento de Matemática, Facultad de Ciencias Exactas y Naturales, UniversiDad de Buenos Aires, 1428 Buenos Aires, Argentina

E-mail address: gacosta@dm.uba.ar

Departamento de Matemática, Facultad de Ciencias Exactas y Naturales, UniversiDad de Buenos Aires, 1428 Buenos Aires, Argentina

E-mail address: garmenta@dm.uba.ar 